O. lliev, G. Printsypar, S. Rief

A two-dimensional model of the pressing section of a paper machine including dynamic capillary effects 
(C) Fraunhofer-Institut für Techno- und Wirtschaftsmathematik ITWM 2012

ISSN 1434-9973

Bericht 211 (2012)

Alle Rechte vorbehalten. Ohne ausdrückliche schriftliche Genehmigung des Herausgebers ist es nicht gestattet, das Buch oder Teile daraus in irgendeiner Form durch Fotokopie, Mikrofilm oder andere Verfahren zu reproduzieren oder in eine für Maschinen, insbesondere Datenverarbeitungsanlagen, verwendbare Sprache zu übertragen. Dasselbe gilt für das Recht der öffentlichen Wiedergabe.

Warennamen werden ohne Gewährleistung der freien Verwendbarkeit benutzt.

Die Veröffentlichungen in der Berichtsreihe des Fraunhofer ITWM können bezogen werden über:

Fraunhofer-Institut für Techno- und Wirtschaftsmathematik ITWM Fraunhofer-Platz 1

67663 Kaiserslautern

Germany

Telefon: $\quad+49(0) 631 / 31600-4674$

Telefax: $\quad+49(0) 631 / 31600-5674$

E-Mail: presse@itwm.fraunhofer.de

Internet: www.itwm.fraunhofer.de 
Das Tätigkeitsfeld des Fraunhofer-Instituts für Techno- und Wirtschaftsmathematik ITWM umfasst anwendungsnahe Grundlagenforschung, angewandte Forschung sowie Beratung und kundenspezifische Lösungen auf allen Gebieten, die für Techno- und Wirtschaftsmathematik bedeutsam sind.

In der Reihe »Berichte des Fraunhofer ITWM « soll die Arbeit des Instituts kontinuierlich einer interessierten Öffentlichkeit in Industrie, Wirtschaft und Wissenschaft vorgestellt werden. Durch die enge Verzahnung mit dem Fachbereich Mathematik der Universität Kaiserslautern sowie durch zahlreiche Kooperationen mit internationalen Institutionen und Hochschulen in den Bereichen Ausbildung und Forschung ist ein großes Potenzial für Forschungsberichte vorhanden. In die Berichtreihe werden sowohl hervorragende Diplom- und Projektarbeiten und Dissertationen als auch Forschungsberichte der Institutsmitarbeiter und Institutsgäste zu aktuellen Fragen der Techno- und Wirtschaftsmathematik aufgenommen.

Darüber hinaus bietet die Reihe ein Forum für die Berichterstattung über die zahlreichen Kooperationsprojekte des Instituts mit Partnern aus Industrie und Wirtschaft.

Berichterstattung heißt hier Dokumentation des Transfers aktueller Ergebnisse aus mathematischer Forschungs- und Entwicklungsarbeit in industrielle Anwendungen und Softwareprodukte - und umgekehrt, denn Probleme der Praxis generieren neue interessante mathematische Fragestellungen.

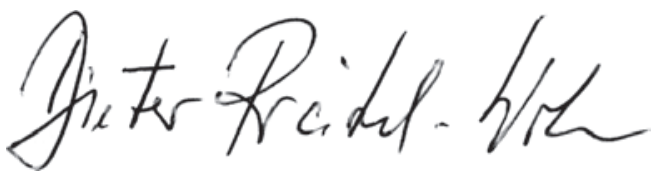

Prof. Dr. Dieter Prätzel-Wolters Institutsleiter

Kaiserslautern, im Juni 2001 



\title{
A Two-Dimensional Model of the Pressing Section of a Paper Machine Including Dynamic Capillary Effects
}

\author{
O. Iliev · G. Printsypar · S. Rief
}

\begin{abstract}
The paper production is a problem with significant importance for the society and it is a challenging topic for scientific investigations. This study is concerned with the simulations of the pressing section of a paper machine. A two-dimensional model is developed to account for the water flow within the pressing zone. Richards' type equation is used to describe the flow in the unsaturated zone. The dynamic capillary pressure-saturation relation proposed by Hassanizadeh and co-workers (Hassanizadeh et al., 2002; Hassanizadeh, Gray, 1990, 1993a) is adopted for the paper production process.

The mathematical model accounts for the co-existence of saturated and unsaturated zones in a multilayer computational domain. The discretization is performed by the MPFA-O method. The numerical experiments are carried out for parameters which are typical for the production process. The static and dynamic capillary pressure-saturation relations are tested to evaluate the influence of the dynamic capillary effect.
\end{abstract}

Keywords two-phase flow in porous media · steady modified Richards' equation · finite volume method $\cdot$ dynamic capillary pressure $\cdot$ pressing section of a paper machine $\cdot$ multipoint flux approximation

Iliev, O., Printsypar, G., Rief, S.

Department of Flow and Material Simulation,

Fraunhofer Institute for Industrial Mathematics (ITWM),

Fraunhofer-Platz 1, D-67663 Kaiserslautern, Germany

Iliev, O., Printsypar, G.

Technical University Kaiserslautern,

Postfach 3049

D-67653 Kaiserslautern

Iliev, O.

E-mail: iliev@itwm.fraunhofer.de

Printsypar, G.

E-mail: printsyp@itwm.fraunhofer.de

Rief, S.

E-mail: rief@itwm.fraunhofer.de 


\section{Introduction}

The paper production is an industrial applications, which attracts attention of many scientists. It is a challenging problem, investigated from different points of view by scientists from different fields. We are concerned with the mathematical modeling and simulation of the pressing section of a paper machine.

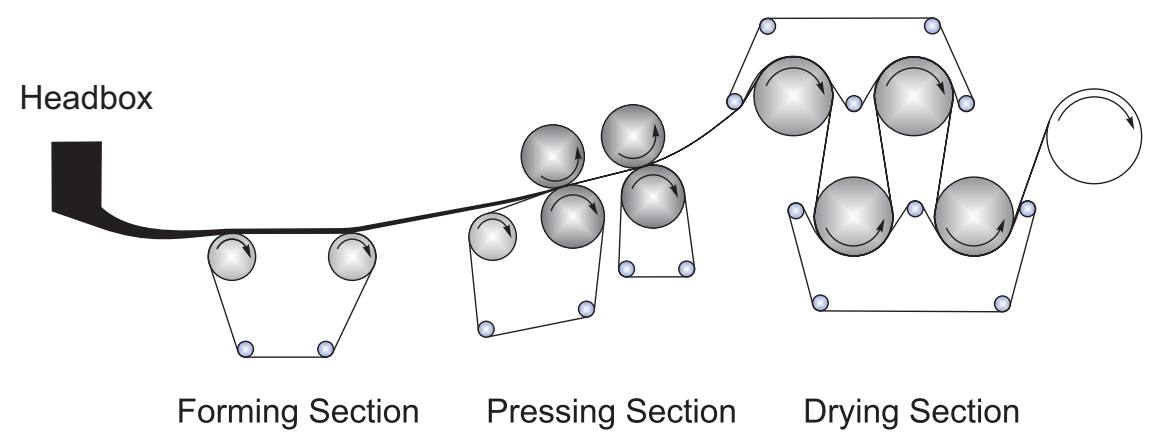

Fig. 1 Schematic representation of a paper machine

\subsection{Overview on the paper machine}

The paper machine is a huge piece of equipment which typically consists of four main parts (see Fig. 1): the headbox, the forming section, the pressing section and the drying section (see Metso Corporation (2010); Paper academy (2011)). Special woven plastic fabric meshes, so-called conveyor belts, are used to transport the paper through all sections of the paper machine. During the production process, a wood pulp is transformed into a final paper product by performing different dewatering techniques. The headbox provides the suspension which consists of $99 \%$ of water and $1 \%$ of solid phase, wooden fibers. In the forming section, dewatering is performed by the natural filtration and sometimes with the help of suction boxes. After the forming section, the dry solid content of the paper increases to about $20 \%$. In the next pressing section, the dewatering is carried out by a mechanical pressing of the paper layer against properly selected fabrics, so-called felts. The simplest construction of a pressing nip consists of two rotating rolls with the paper-felt sandwich transported between them at high speed up to $2000 \mathrm{~m} / \mathrm{min}$ as shown in Fig. 2 on the left. There exists also another type of a pressing nip which is called shoe press (see Fig. 2 on the right). The advantage of the shoe press is an extended pressing zone, which is about $300 \mathrm{~mm}$ in comparison with $40 \mathrm{~mm}$ in the roll press. In contrast, the thickness of the paper-felt sandwich is about $4 \mathrm{~mm}$ and the thickness of the paper layer can go down to 100 micrometers. During the pressing of the paper layer against the felts, water is squeezed out of the paper and enters the felts. So the water content of the paper decreases to about $50 \%$ after the pressing section. The last section is the drying section where the remaining water is removed by evaporation. Paper is 
transported over streamheated cylinders and comes out of the drying section with a water content of $5 \%$.

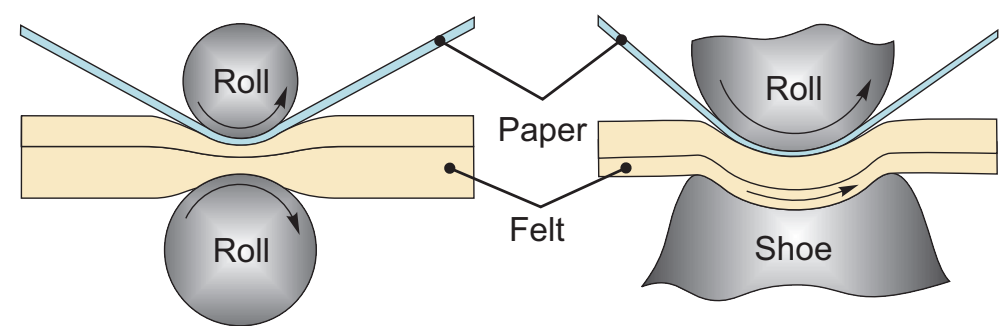

Fig. 2 Pressing nips: roll press (on the left), shoe press (on the right)

The pressing is a more economic way to remove the water from the paper than the drying. Therefore, the industry is actively working on improving the dewatering in the pressing section. The laboratory experiments for the paper machine are very expensive and difficult to carry out. The simulation approach allows to reduce time and money needed for improving the design of the pressing section.

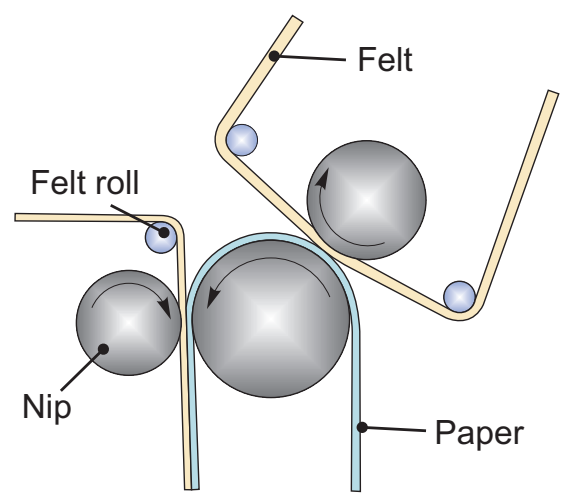

Fig. 3 Pressing section

The pressing section is composed of a sequence of rolls and typically one shoe. Their positioning may vary depending on a paper machine. Fig. 3 shows a sketch of the pressing section. The paper web is usually transported either on one felt in the top or bottom position or between two felts as a sandwich. In some cases, when the paper web is strong enough compared with the applied stress in operation, the web is transported towards the next press nip or to the dryer section without any felt support (Paper academy, 2011). Thus, the paper layer sometimes is in contact with the felt and sometimes separated from it while passing the pressing section. Our mathematical model of the pressing section considers the layers to be transported all together. The separation is taken into account by specifying no-flow boundary conditions on the parts of the interfaces where the layers are not in contact in reality. 
1.2 State of the art for the pressing section modeling

The pressing process in a paper machine is very complex since such features as moving and deformable porous media, computational domain composed from different layers, multiphase flow, etc. have to be taken into account. There exist various approaches to model the pressing section of a paper machine (Bezanovic et al., 2006, 2007a,b; Hiltunen, 1995; Kataja et al., 1992). The mass and momentum conservation equations are used together with a Lagrangian formulation along displacement characteristic lines (solid flow lines) in Hiltunen (1995); Kataja et al. (1992). In Bezanovic et al. (2006, 2007a,b) the Lagrangian formulation of mass balance is used. In the last work by Bezanovic et al. (2007b) the compressible air is also considered. But all these models have a common feature, which is neglecting the capillary forces. Models which take into account the capillary effect are presented in Bermond (1997); Rief (2005, 2007); Velten, Best (2000). The model described by Bermond (1997) uses a two-phase flow model including capillary pressure-saturation relation and introduces thermal aspects. In Rief (2005, 2007); Velten, Best (2000), the Richards approach for flow in unsaturated porous media is adopted. None of the above mentioned models considers the dynamic capillary pressure effect, which is our main target. Further on, advanced finite volume discretization, namely MPFA-O method, is employed here in order to provide more accurate discretization. As a starting point, we have chosen the model realized in Rief $(2005,2007)$.

\subsection{Introduction to capillary effects}

Typically, the capillary effect has a significant influence on the modeling of multiphase flow in porous media (see Bear (1972); Bear, Bachmat (1990); Bear, Verruijt (1987); Helmig (1997)). The capillary pressure is defined as the difference in the phase pressures:

$$
p_{c}=p_{n}-p_{w}
$$

where $p_{n}$ and $p_{w}$ are the pressures of non-wetting and wetting phases, respectively. To include this effect in numerical experiments, the capillary pressure can be presented as a function of the water saturation, and sometimes of other parameters of the filtration process. The typical approach to obtain this function is to construct the capillary pressure-saturation relation based on laboratory experiments. This process is carried out in the following way. To construct for example a drainage curve, at the beginning the sample of a porous medium is fully saturated with water. Then, air starts infiltrating the sample by increasing its pressure stepwise. When equilibrium is reached, the capillary pressure and the water saturation are measured. This measurement forms one point at the targeted capillary pressure-saturation curve. The time which is needed to reach equilibrium after changing the pressure can take from several hours to several days. Construction of the complete capillary pressuresaturation curve for the felt, which is used in the paper production process, may take several days.

Many scientists worked on parametrizing the measurement results (e.g. see Broocks, Corey (1964); Leverett (1941); Van Genuchten (1980)). This approach works quite accurately in case of slow infiltration processes. In our case, the drying process of the paper pulp takes much less time than the construction of the static capillary 
pressure-saturation curve. There also exist different studies which try to understand and parametrize a dynamic capillary pressure which is not based on the equilibrium condition (see Barenblatt et al. (1987, 2002); Bourgeat, Panfilov (1998); Kalaydjian (1992); Ross (2000); Hassanizadeh et al. (2002); Hassanizadeh, Gray (1990, 1993a)). The detailed overview and analysis of these models was done by Manthey (2006). We have chosen the approach proposed by Hassanizadeh, Gray (1990). Their method was derived based on the physical aspects of the porous media flow. Adaptation of this model to processes in the pressing section, as well as performing computational experiments for evaluation of the influence of the dynamic capillary pressure, are the main topics of this paper.

\subsection{Discretization methods}

The model of the pressing section has several specific features which have to be taken into account when we choose a discretization method. First of all, we would like to preserve boundaries between layers during discretization. Therefore, a grid which is based on the solid deformations is used. It means that we deal with a quadrilateral nonorthogonal grid. Moreover, the layered domain leads to discontinuities in permeability. In spite of it, the continuity of the pressure and the fluxes at local physical interfaces between grid cell has to be preserved. We also have to take into account that the permeability is presented by a full tensor and not by a diagonal one.

A number of schemes were proposed recently to discretize such kind of problems (see Aavatsmark (2002, 2007); Edwards (2002); Herbin and Hubert (2008) and references therein). Some of them were tested by Herbin and Hubert (2008) for various types of test problems. They concluded that there does not exist the best scheme for any problem and that the method has to be chosen taking into account the specific features of the considered problem. Our choice is the MPFA-O method (see Aavatsmark (2002, 2007); Eigestad and Klausen (2005)). This method is intuitive. It is simply adopted for the complex boundary and interface conditions which have to be preserved, and its usage for our problems has shown reliable results.

\subsection{Goals and structure of the paper}

The pressing process is carried out at high speeds and the movement of water within the pressing zone cannot be considered as a slow process. The goal of these studies is to include the dynamic capillary effect in the simulation of the pressing section of a paper machine. We develop a two-dimensional mathematical model which adopts the dynamic capillary pressure-saturation relation proposed by Hassanizadeh and co-workers. Section 2 describes the development of the mathematical model which accounts for all specific features of this problem. The discretization is presented in Section 3. The numerical experiments which evaluate the influence of the dynamic capillary effect are developed in Section 4. Finally, we draw conclusions in Section 5.

\section{Mathematical Model}

In this study we are concerned with the two-dimensional model for the pressing section of a paper machine. Let us assume that the paper-felt sandwich is transported 
through the press nips from the left to the right with velocity $\mathbf{V}_{s, i n}$, as indicated in Fig. 4. The horizontal machine direction is designated as $x$-direction. The vertical component is the $z$-direction. Since the length of the cylindrical rolls is large lateral boundary effects are not considered. Hence, the $y$-direction is neglected. A computational domain $\Omega \subset \mathbb{R}^{2}$ is introduced as indicated in Fig. 4 . The boundaries of the domain $\Omega$ are defined as $\partial \Omega=\Gamma_{L} \cup \Gamma_{U} \cup \Gamma_{R} \cup \Gamma_{D}$.

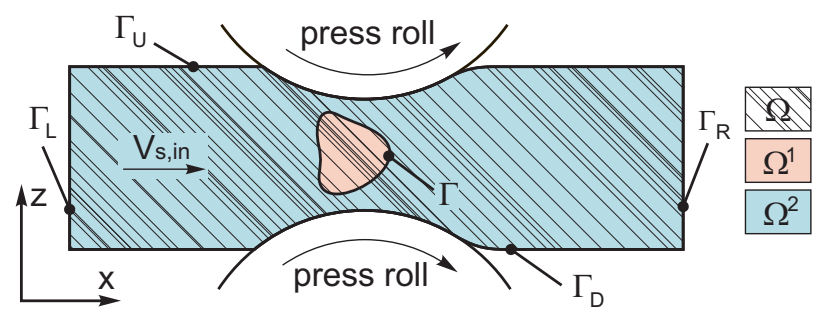

Fig. 4 Computational domain with two flow regimes

One of the main issues in the modeling of the pressing section is to account for fully saturated regions, which appear within the pressing zone. So, one has to distinguish between two different flow regimes: single-phase water flow and twophase air-water flow. Therefore, the computational domain $\Omega$ is divided into two nonoverlapping subdomains $\Omega^{\alpha}$ with $\alpha$-phase flow for $\alpha=1,2$ as shown in Fig. 4. We denote the interface between these domains as $\Gamma=\overline{\Omega^{1}} \cap \overline{\Omega^{2}}$. It is unknown in advance, and finding $\Gamma$ is a part of the solution procedure. $\Omega^{1}$ could even be the empty set.

In this section we are going to present mathematical models for both flow regimes taking into account layered porous medium (see Bear (1972); Bear, Bachmat (1990); Bear, Verruijt (1987); Helmig (1997)). At first, we are going to introduce a mathematical model for the single-layer case. Then, the mathematical model will be extended to the multilayer case. Concluding this section, we will briefly describe elasticity model, which is used to resolve the solid deformations.

Before we start formulating model equations let us make some assumptions.

Assumption 1 (Richards' assumption) Within the computational domain, the air remains at atmospheric pressure.

Assumption 2 Gravity is negligible.

Assumption 3 All phases are incompressible.

Assumption 1 is made to simplify the mathematical model. But the admissibility of this statement still has to be shown and will be investigated in our future work. Assumption 2 is reasonable since the capillary and external forces are dominant in the pressing process. Therefore, the gravity does not significantly influence the movement of water inside the computational domain. Assumption 3 obviously makes sense for the water and solid phases. In case of the air phase, it still has to be confirmed. 
2.1 Single-phase water flow

Water flow within a porous medium is modeled by the mass conservation equation without sources and sinks:

$$
\frac{\partial\left(\phi \rho_{w}\right)}{\partial t}+\operatorname{div}\left(\phi \rho_{w} \mathbf{V}_{w}\right)=0, \quad \mathbf{x} \in \Omega^{1}
$$

where $\phi([-])$ is the porosity, $\rho_{w}$ is the density of water measured in $\left[\mathrm{kg} / \mathrm{m}^{3}\right], t$ is the time in $[s], \mathbf{V}_{w}$ is the velocity of water in $[m / s]$. Let us also remark that in the following all vectors and tensors will be written in bold type. To define the water velocity $\mathbf{V}_{w}$ we use the momentum equation for the water phase, which can be presented by Darcy's law:

$$
\phi\left(\mathbf{V}_{w}-\mathbf{V}_{s}\right)=-\frac{\mathbf{K}}{\mu_{w}} \operatorname{grad} p_{w}, \quad \mathbf{x} \in \Omega^{1}
$$

where $\mathbf{V}_{s}$ is the velocity of the solid in $[\mathrm{m} / \mathrm{s}], \mu_{w}$ is the viscosity of the water in $[\mathrm{Pas}], \mathbf{K}$ is the intrinsic permeability tensor in $\left[\mathrm{m}^{2}\right], p_{w}$ is the pressure of water in $[P a]$.

We set the partial derivative w.r.t. time in (1) to zero since we are interested in a steady-state solution. Taking into account Assumption 3, which states that the water phase is incompressible, and combining equations (1) and (2), we obtain:

$$
-\operatorname{div}\left(\frac{\mathbf{K}}{\mu_{w}} \operatorname{grad} p_{w}\right)+\operatorname{div}\left(\phi \mathbf{V}_{s}\right)=0, \quad \mathbf{x} \in \Omega^{1} .
$$

The distribution of the water pressure within $\Omega^{1}$ is governed by equation (3).

\subsection{Two-phase air-water flow}

To model the flow of air and water inside a porous medium we use Richards' approach (see Assumption 1). Then, the mass conservation equation for water phase yields:

$$
\frac{\partial\left(\phi S \rho_{w}\right)}{\partial t}+\operatorname{div}\left(\phi S \rho_{w} \mathbf{V}_{w}\right)=0, \quad \mathbf{x} \in \Omega^{2}
$$

where $S([-])$ is the saturation of the water phase. The generalized Darcy's law in the case of the two-phase flow takes the form:

$$
\phi S\left(\mathbf{V}_{w}-\mathbf{V}_{s}\right)=-\frac{k_{r w}}{\mu_{w}} \mathbf{K} \operatorname{grad} p_{w}, \quad \mathbf{x} \in \Omega^{2} ;
$$

where $k_{r w}([-])$ is the relative permeability of the water phase.

We have to supplement equations (4) and (5) with a capillary pressure-saturation relation. The drying in the pressing section is a fast dynamic process. Therefore, we decided to include the dynamic capillary effect. We adopt the model derived by Hassanizadeh and Gray to the pressing process and obtain:

$$
p_{w}+p_{c}^{\text {stat }}=\tau \frac{D^{s} S}{D t}, \quad \mathbf{x} \in \Omega^{2}
$$


where $p_{c}^{\text {stat }}$ is the empirical static capillary pressure-saturation relation, $\tau$ is a socalled material coefficient in $[P a s], D^{s} S / D t$ is the material derivative w.r.t. a reference frame fixed to the solid phase:

$$
\frac{D^{s} S}{D t}=\frac{\partial S}{\partial t}+\mathbf{V}_{s} \cdot \operatorname{grad} S
$$

Let us remark that the material coefficient $\tau$ may be a function of saturation and other parameters, but in these work we consider $\tau$ to be a constant. We also notice that the case $\tau=0$ leads to the model with static capillary pressure-saturation relation.

In case of the steady-state process, equations (4)-(7) yield:

$$
\begin{array}{r}
-\operatorname{div}\left(\frac{k_{r w}}{\mu_{w}} \mathbf{K} \operatorname{grad} p_{w}\right)+\operatorname{div}\left(\phi S \mathbf{V}_{s}\right)=0, \quad \mathbf{x} \in \Omega^{2} ; \\
p_{w}+p_{c}^{\text {stat }}=\tau \mathbf{V}_{s} \cdot \operatorname{grad} S, \quad \mathbf{x} \in \Omega^{2} .
\end{array}
$$

\subsection{Interfacial conditions}

On the interface $\Gamma$ between domains with single-phase and two-phase flows we have to satisfy some continuity conditions. At first, let us introduce an operator $[f]_{\Gamma}$ which indicates a jump of the function $f$ across the interface $\Gamma$ :

$$
[f]_{\Gamma}=\lim _{t \rightarrow \Gamma+0} f(t)-\lim _{t \rightarrow \Gamma-0} f(t) .
$$

Then, continuity of water pressure and continuity of normal fluxes are imposed:

$$
\left[p_{w}\right]_{\Gamma}=0, \quad\left[\mathbf{J}_{w} \cdot \mathbf{n}\right]_{\Gamma}=0
$$

where $\mathbf{n}$ is the unit normal vector to $\Gamma, \mathbf{J}_{w}$ is the water flux, which is defined as:

$$
\mathbf{J}_{w}=\left\{\begin{array}{l}
-\frac{\mathbf{K}}{\mu_{w}} \operatorname{grad} p_{w}+\phi \mathbf{V}_{s}, \text { for all } \mathbf{x} \in \overline{\Omega^{1}} \\
-\frac{k_{r w}}{\mu_{w}} \mathbf{K} \operatorname{grad} p_{w}+\phi S \mathbf{V}_{s}, \text { for all } \mathbf{x} \in \Omega^{2}
\end{array}\right.
$$

2.4 Full model

Summarizing both flow models, we want to reformulate the problem $(3),(8),(9),(11),(12)$ in a more suitable way for further developments. Let us make the following assumption:

Assumption $4 k_{r w} \in C\left(\left[S_{r}, 1\right]\right), k_{r w}:\left[S_{r}, 1\right] \rightarrow\left[k_{*}, 1\right]$ is an increasing function, where $k_{*}>0 \in \mathbb{R}$ and $S_{r}>0 \in \mathbb{R}$ is the residual saturation ([-]).

Taking into account Assumption 4 we rewrite equations (3),(8),(9) in the following form:

$$
\begin{aligned}
& -\operatorname{div}\left(\frac{k_{r w}}{\mu_{w}} \mathbf{K} \operatorname{grad} p_{w}\right)+\operatorname{div}\left(\phi S \mathbf{V}_{s}\right)=0, \quad \mathbf{x} \in \Omega, \\
& S=1, \quad \mathbf{x} \in \Omega^{1}, \\
& p_{w}+p_{c}^{\text {stat }}=\tau \mathbf{V}_{s} \cdot \operatorname{grad} S, \quad \mathbf{x} \in \Omega^{2} ;
\end{aligned}
$$


where we assume that $k_{r w}=k_{r w}(S), \mathbf{K}=\mathbf{K}(\mathbf{x}), \phi=\phi(\mathbf{x}), \mathbf{V}_{s}=\mathbf{V}_{s}(\mathbf{x}), p_{c}^{\text {stat }}=$ $p_{c}^{\text {stat }}(S, \phi), \tau=\tau(\mathbf{x})$.

We notice that equation (13) coincides with (3) in $\Omega^{1}$ and with (8) in $\Omega^{2}$. We also have to make sure that continuity conditions (11), (12) are satisfied in this case. Continuity of the water pressure $p_{w}$ follows from the definition of the nonlinear convection-diffusion equation (13). Continuity of the normal fluxes follows directly from integration of equation (13) over a small neighborhood of the boundary $\Gamma$.

\subsection{Layered computational domain}

In general, the computational domain $\Omega$ consists of several layers (see Fig. 5). Therefore, it is divided into nonoverlapping subdomains $\Omega_{1}, \Omega_{2}, \ldots, \Omega_{L}$, where $L$ is the total number of layers. Interfaces between the subdomains are denoted by $\Gamma_{l}=\bar{\Omega}_{l} \cap \bar{\Omega}_{l+1}$ for all $l=\overline{1, L-1}$.

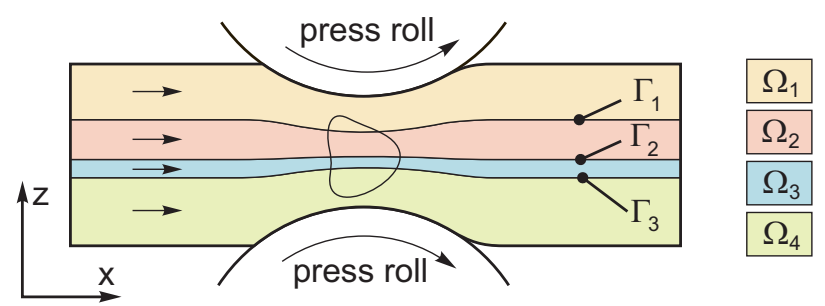

Fig. 5 Computational domain with layers

Then, the system of equations (13)-(15) has to be satisfied together with continuity of pressure and continuity of normal fluxes on the interfaces between layers:

$$
\left[p_{w}\right]_{\Gamma_{l}}=0, \quad\left[\mathbf{J}_{w} \cdot \mathbf{n}\right]_{\Gamma_{l}}=0, \quad l=\overline{1, L-1}
$$

where we remember that each layer has its own properties, therefore, functions $k_{r w}=$ $k_{r w}(S, \mathbf{x}), \mathbf{K}=\mathbf{K}(\mathbf{x}), \phi=\phi(\mathbf{x}), p_{c}^{\text {stat }}=p_{c}^{\text {stat }}(S, \phi, \mathbf{x}), \tau=\tau(\mathbf{x})$ may have jumps over the layer interfaces.

\subsection{Boundary conditions}

To close the system of equations (13)-(16) we impose boundary conditions. At first let us make an assumption.

Assumption 5 Boundaries $\Gamma_{L}$ and $\Gamma_{R}$ are far away from the pressing zone.

On the left boundary $\Gamma_{L}$ the distributions of saturation and pressure are known. This case is typical for the production process. Then, Dirichlet boundary conditions are imposed on $\Gamma_{L}$. Assumption 5 means that water remains at equilibrium w.r.t. the solid skeleton on $\Gamma_{L}$ and the dynamic effect is absent there. Therefore, for the pressure we use the dependence $p_{c}^{\text {stat }}$ on initial values of saturation. Since the right boundary $\Gamma_{R}$ is also far from the pressing zone, it is assumed that the water reaches 
the equilibrium state w.r.t. the solid skeleton on $\Gamma_{R}$. Therefore, we apply no-flow boundary conditions on $\Gamma_{R}$. On the upper and lower boundaries $\Gamma_{U}$ and $\Gamma_{D}$ we assume that there is no escape of water and also impose zero Neumann boundary conditions. Hence, we have:

$$
\begin{aligned}
& \left.S\right|_{\Gamma_{L}}=C_{0}(\mathbf{x}),\left.\quad p_{w}\right|_{\Gamma_{L}}=-p_{c}^{\text {stat }}\left(C_{0}\right) ; \\
& \left.\left(-\frac{k_{r w}}{\mu_{w}} \mathbf{K} \operatorname{grad} p_{w}\right) \cdot \mathbf{n}_{s}\right|_{\Gamma_{R}}=0 ; \\
& \left.\left(-\frac{k_{r w}}{\mu_{w}} \mathbf{K} \operatorname{grad} p_{w}\right) \cdot \mathbf{n}\right|_{\Gamma_{U}, \Gamma_{D}}=0 ;
\end{aligned}
$$

where $\mathbf{n}_{s}$ is the unit vector collinear to $\mathbf{V}_{s}$. We remark that the second term of water flux related to convection in (19) is equal to zero since $\mathbf{V}_{s} \cdot \mathbf{n}=0$ for the outer unit normal vector $\mathbf{n}$ to $\Gamma_{U}$ or $\Gamma_{D}$.

According to the production process, sometimes layers of the paper and felt in the paper-felt sandwich separate as shown in Fig. 2, 3 (see Section 1.1). To take it into account we also provide a possibility to impose no-flow boundary conditions on some parts of the interfaces between layers.

\subsection{Elasticity model}

The presented flow model has to be supplemented by an elasticity model, which accounts for the solid deformations. In the current work we use developments from Rief (2005). He simulated the pressing section considering the elasticity model weakly coupled with the flow model supplemented by static capillary pressure-saturation relation. For the completeness of the stated model let us recall the elasticity model from Rief (2005, 2007).

The main reason of the solid deformations is the pressing forces which are applied to the paper-felt sandwich. These forces are very large, a typical value is about $100 \mathrm{kN} / \mathrm{m}$ in the roll press and about $1000 \mathrm{kN} / \mathrm{m}$ in the shoe press. Under these conditions the solid deformations caused by forces of water acting on the solid phase can be neglected in a first approximation. The solid phase is assumed to be incompressible and the porous medium gets deformed as a rearrangement of the solid skeleton in vertical direction. According to Velten, Best (2000); Jewett et al. (1980), the felt and the paper are assumed to behave viscoelastically. Since the paper-felt sandwich is transported in machine $x$-direction, we state the Kelvin-Voigt model for $L$ layers:

$$
\begin{aligned}
& \mathrm{t}(x)=E_{1}\left(\varepsilon_{1}(x)\right)+\Lambda_{1} c \frac{d}{d x} E_{1}\left(\varepsilon_{1}(x)\right)-k \mathrm{t}_{\max }(x), \\
& \mathrm{t}(x)=E_{i}\left(\varepsilon_{i}(x)\right)+\Lambda_{i} c \frac{d}{d x} E_{i}\left(\varepsilon_{i}(x)\right), \quad i=\overline{2, L}
\end{aligned}
$$

where $\mathrm{t}$ is the stress measured in $[\mathrm{Pa}]$. The dimensionless strain is defined by

$$
\varepsilon_{i}(x)=\frac{l_{0, i}-l_{i}(x)}{l_{0, i}} \text { for each layer } i=\overline{1, L},
$$

with undeformed and deformed thicknesses of the layer $i$ at coordinate $x$ denoted by $l_{0, i}(x)$ and $l_{i}(x)$, respectively. In general, $E_{i}$ is some nonlinear function related to the 
elastic part of the stress and the strains. $\Lambda_{i}([s])$ is the viscoelastic time constant, which determines the speed of relaxation. The constant $c$ is the absolute value of the velocity $\mathbf{V}_{s, i n}$.

Equations (21) correspond to the felts. Equation (20) corresponds to the paper layer and has an additional third term on the right hand side. This term is introduced to model the permanent compression, which appears due to plasticity of the paper. We assume that the value of the permanent deformation depends linearly on the maximum stress to which the paper has been exposed multiplied by some constant $k$ :

$$
\mathrm{t}_{\text {max }}\left(x_{0}\right)=\max _{x \leq x_{0}} \mathrm{t}(x) .
$$

To close the system of equations $(20),(21)$ we also use the following relation:

$$
\sum_{i=1}^{L} \varepsilon_{i}(x) l_{0, i}=l_{0}-f(x),
$$

where $l_{0}=\sum_{i=1}^{L} l_{0, i}$ is the total thickness of the undeformed paper-felt sandwich. Due to the fact that the thickness of the paper-felt sandwich will never exceed $l_{0}$, the function $f(x)$ has the form:

$$
f(x)=\min \left\{l_{0} \text {, distance between press profiles at position } x\right\} .
$$

To resolve the system of equations $(20),(21),(24)$ one more input parameter has to be provided. The first possibility is to provide the minimum distance between press profiles, which defines the position of the pressing nips and the geometry of the computational domain $\Omega$. Another possibility which is more convenient for the industrial applications is to define the pressing force, which is equal to the integral of the stress profile over the length of the computational domain. Having one of these parameters, the system of equations can be solved.

After we find the distribution of the stress and the strains, it is possible to compute the necessary input data for the flow solver. Since the thickness of the layers is small we consider that the porosity changes only in horizontal direction. Then, the porosity for each layer can be found as:

$$
\phi_{i}(x)=\frac{\varepsilon_{i}(x)+\phi_{0, i}}{\varepsilon_{i}(x)+1} \text { for all } i=\overline{1, L},
$$

where $\phi_{0, i}$ is the porosity of the $i$ th undeformed layer. Using the computed strains, the flow mesh can be obtained immediately as well as the distribution of the solid velocity $\mathbf{V}_{s}(\mathbf{x})$ (for more details see Rief $(2005,2007)$ ).

Remark 1 As it was mentioned in the introduction, we also consider the second type of the press nips, so-called shoe press. In this case the paper-felt sandwich is not transported strictly in horizontal direction (see Fig. 2). But since the thickness of the pressing zone is very small compared to its length the angle between the paper-felt sandwich and machine direction is small. Therefore, the assumption on the horizontal transportation is still a very good approximation, and we use the same elasticity model for the shoe press.

More detailed discussions on this elasticity model, its discretization and solution can be found in Rief $(2005,2007)$. 


\section{Discretization}

Let us now discuss the discretization on a quadrilateral unstructured grid of the flow model stated in the previous section. At first the mesh is introduced.

Definition 1 Let $\Omega$ be an open bounded polygonal subset of $\mathbb{R}^{2}$ with boundary $\partial \Omega$. The discretization of $\Omega$ is defined as $\mathcal{D}=(\mathcal{T}, \mathcal{E}, \mathcal{X})$, where the following holds.

$-\mathcal{T}$ is the finite set of nonoverlapping quadrilateral cells $\mathcal{K}$ ('control volumes') such that $\bar{\Omega}=\cup_{\mathcal{K} \in \mathcal{T}} \overline{\mathcal{K}}$. The boundary of each control volume is denoted by $\partial \mathcal{K}=\overline{\mathcal{K}} \backslash \mathcal{K}$.

$-\mathcal{E}$ is the finite set of one-dimensional edges of all control volumes. For any control volume $\mathcal{K} \in \mathcal{T}$ there exists a subset $\mathcal{E}_{\mathcal{K}}$ of $\mathcal{E}$ such that $\partial K=\cup_{\sigma \in \mathcal{E}_{\mathcal{K}}} \bar{\sigma}$. Furthermore, $\mathcal{E}=\cup_{\mathcal{K} \in \mathcal{T}} \mathcal{E}_{\mathcal{K}}$. For any $\mathcal{K}, \mathcal{L}$ from $\mathcal{T}$ with $\mathcal{K} \neq \mathcal{L}$, either $\overline{\mathcal{K}} \cap \overline{\mathcal{L}}=\emptyset$ or $\overline{\mathcal{K}} \cap \overline{\mathcal{L}}=\bar{\sigma}$ for some $\sigma \in \mathcal{E}$, which then will be denoted by index $\mathcal{K} \mid \mathcal{L}$.

$-\mathcal{X}=\left(\mathbf{x}_{\mathcal{K}}\right)_{\mathcal{K} \in \mathcal{T}}$ is the finite set of points of $\Omega$ ('cell centers') such that $\mathbf{x}_{\mathcal{K}} \in \mathcal{K}$ for all $\mathcal{K} \in \mathcal{T}$.

Remark 2 In the previous section the computational domain $\Omega$ was introduced. In Definition 1 the polygonal set still denoted by $\Omega$ is an approximation of the original computational domain.

Definition 1 introduces some general notations for the mesh which is used for discretization. The mesh which is constructed for our computational domain has constant step size $h_{x}$ in $x$-direction (see Fig. 6). In $z$-direction at the left and right boundaries where no deformations occur the mesh has also constant step size $h_{z}$. If the cell contains an interface between two layers the step size $h_{z}$ is divided into two parts to resolve the interface. In general, the mesh has varying step size in $z$ direction which is proportional to the solid deformations. Cell center $\mathbf{x}_{\mathcal{K}}$ is defined as the intersection point of intervals connecting midpoints of the opposed edges of the control volume $\mathcal{K}$.

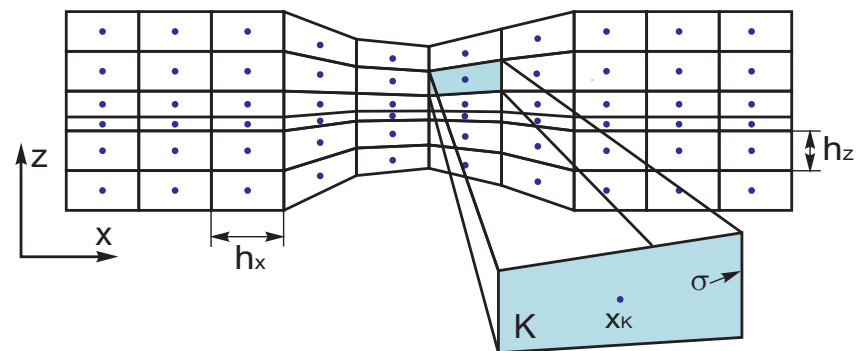

Fig. 6 Discretization of the computational domain

The system of equations (13)-(15) together with interfacial conditions (16) and boundary conditions (17)-(19) is discretized with the help of the finite volume method (see e.g. Eymard et al. (2006)). To simplify the notations we omit the index ' $w$ ' in the variables $p_{w}, k_{r w}$ and $\mu_{w}$. 
Now let us introduce some notations. If $\sigma=\sigma_{\mathcal{K} \mid \mathcal{L}}$ is the common edge of cells $\mathcal{K}$ and $\mathcal{L}$ then we denote:

$$
\begin{aligned}
S_{\sigma} & =\frac{1}{2}\left(S_{\mathcal{K}}+S_{\mathcal{L}}\right) \\
S_{\sigma,+} & =\left\{\begin{aligned}
S_{\mathcal{K}}, & \text { if } \mathbf{V}_{s} \cdot \mathbf{n}_{\sigma} \geq 0 \\
S_{\mathcal{L}}, & \text { if } \mathbf{V}_{s} \cdot \mathbf{n}_{\sigma}<0
\end{aligned}\right.
\end{aligned}
$$

where $S_{\mathcal{K}}$ is the approximated value of $S$ at $\mathbf{x}_{\mathcal{K}}, \mathbf{n}_{\sigma}$ is the normal unit vector to $\sigma$ outward to $\mathcal{K}$.

Integrating (13) over the control volume $\mathcal{K}$, we obtain:

$$
-\sum_{\sigma \in \mathcal{E}_{\mathcal{K}}} \frac{k_{r}\left(S_{\sigma}\right)}{\mu} F_{\mathcal{K}, \sigma}+\sum_{\sigma \in \mathcal{E}_{\mathcal{K}}} m_{\sigma} \phi_{\sigma} S_{\sigma,+} \mathbf{V}_{s} \cdot \mathbf{n}_{\sigma}=0, \quad \mathcal{K} \in \mathcal{T}
$$

where $m_{\sigma}$ is the one-dimensional measure of the boundary $\sigma, \phi_{\sigma}$ is the porosity at $\sigma$. The general form of $F_{\mathcal{K}, \sigma}$ is:

$$
F_{\mathcal{K}, \sigma}=\sum_{\mathcal{L} \in \mathcal{N}_{\mathcal{K}, \sigma}} t_{\mathcal{K}, \sigma}^{\mathcal{L}} p_{\mathcal{L}}
$$

with transmissibility coefficients $t_{\mathcal{K}, \sigma}^{\mathcal{L}}$ and the subset $\mathcal{N}_{\mathcal{K}, \sigma}$ of all control volumes such that:

$$
\mathcal{N}_{\mathcal{K}, \sigma}=\left\{\mathcal{L} \in \mathcal{T}: \quad \sigma \in \mathcal{E}_{\mathcal{K}}, \quad \bar{\sigma} \cap \overline{\mathcal{L}} \neq \emptyset\right\} .
$$

For the quadrilateral grid the set $\mathcal{N}_{\mathcal{K}, \sigma}$ consists of six control volumes as shown in Fig. 7.

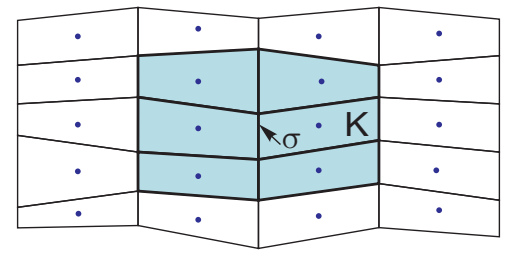

Fig. 7 The set $\mathcal{N}_{\mathcal{K}, \sigma}$ for the quadrilateral grid

The discrete flux $F_{\mathcal{K}, \sigma}$ is an approximation of the integral $\int_{\sigma}\left(\mathbf{n}_{\sigma} \cdot \mathbf{K} \operatorname{grad} p\right) d s$. The main idea of the MPFA method is to obtain the transmissibility coefficients by carrying out some preprocessing calculations, which depend only on the input data. The approximation is carried out by the multipoint flux approximation O-method (see Aavatsmark $(2002,2007)$; Eigestad and Klausen (2005)). Coefficients $t_{\mathcal{K}, \sigma}^{\mathcal{L}}$ are so-called transmissibility coefficients, which satisfy:

$$
\sum_{\mathcal{L} \in \mathcal{N}_{\mathcal{K}, \sigma}} t_{\mathcal{K}, \sigma}^{\mathcal{L}}=0
$$


Finite volume schemes for equations (14) and (15) yield:

$$
\begin{aligned}
& S_{\mathcal{K}}=1, \mathcal{K} \in \mathcal{T}_{1}, \\
& m_{\mathcal{K}}\left(p_{\mathcal{K}}+p_{c}^{s t a t}\left(S_{\mathcal{K}}\right)\right)=\tau \sum_{\sigma \in \mathcal{E}_{\mathcal{K}}} m_{\sigma}\left(S_{\sigma,+}-S_{\mathcal{K}}\right) \mathbf{V}_{s} \cdot \mathbf{n}_{\sigma}, \quad \mathcal{K} \in \mathcal{T}_{2},
\end{aligned}
$$

where $m_{\mathcal{K}}$ is the two-dimensional measure of the control volume $\mathcal{K} . \mathcal{T}_{1}$ and $\mathcal{T}_{2}$ are the sets of the control volumes which approximate the domains $\Omega_{1}$ and $\Omega_{2}$, respectively. These sets satisfy $\mathcal{T}_{1} \cap \mathcal{T}_{2}=\emptyset$ and $\mathcal{T}_{1} \cup \mathcal{T}_{2}=\mathcal{T}$.

Let us now take into account the boundary conditions (17)-(19). Let the set $\mathcal{E}$ be divided into five subsets:

$$
\begin{aligned}
\mathcal{E}_{\text {int }} & =\{\sigma \in \mathcal{E}: \sigma \cap \partial \Omega=\emptyset\}, \\
\mathcal{E}_{\text {ext }, \alpha} & =\left\{\sigma \in \mathcal{E}: \sigma \cap \Gamma_{\alpha} \neq \emptyset\right\}, \quad \alpha=\{L, U, R, D\} .
\end{aligned}
$$

In equations (31) and (33) the following relations are used:

- if $\sigma \in \mathcal{E}_{\mathcal{K}} \cap \mathcal{E}_{\text {ext }, L}$ than

$$
S_{\sigma,+}=\left\{\begin{array}{ll}
S_{\mathcal{K}}, & \text { if } \mathbf{V}_{s} \cdot \mathbf{n}_{\sigma} \geq 0 ; \\
C_{0, \sigma}, & \text { if } \mathbf{V}_{s} \cdot \mathbf{n}_{\sigma}<0 ;
\end{array}, \quad S_{\sigma}=\frac{1}{2}\left(S_{\mathcal{K}}+C_{0, \sigma}\right),\right.
$$

where $C_{0, \sigma}$ is the value of $C_{0}$ at $\sigma$;

- if $\sigma \in \mathcal{E}_{\mathcal{K}} \cap \mathcal{E}_{\text {ext }, R}$ than

$$
S_{\sigma,+}=S_{\mathcal{K}}, \quad S_{\sigma}=S_{\mathcal{K}}
$$

We also remark that if $\sigma \in \mathcal{E}_{\mathcal{K}} \cap\left(\mathcal{E}_{\text {ext,U}} \cup \mathcal{E}_{\text {ext,D}}\right)$ than $\mathbf{n}_{\sigma} \cdot \mathbf{V}_{s}=0$ and $F_{\mathcal{K}, \sigma}=0$. So we do not need to define $S_{\sigma}$ and $S_{\sigma,+}$ there. The boundary conditions (17)-(19) also have to be taken into account while computing transmissibility coefficients $t_{\mathcal{K}, \sigma}^{\mathcal{L}}$ (for more details see Aavatsmark $(2002,2007)$ ).

To solve the nonlinear system of equations (29), (32) and (33) the Newton's method is used (for more details see Deuflhard (2004); Kelley (1995)). Remembering that the static capillary pressure-saturation relation depends also on the porosity, initial guesses for pressure and saturation are chosen as:

$$
p_{\mathcal{K}}^{0}=-p_{c}^{\text {stat }}\left(C_{0}\left(\mathbf{x}_{\mathcal{K}, \Gamma_{L}}\right), \phi\left(\mathbf{x}_{\mathcal{K}, \Gamma_{L}}\right)\right), \quad S_{\mathcal{K}}^{0}=\left(p_{c}^{\text {stat }}\right)^{-1}\left(p_{\mathcal{K}}^{0}, \phi\left(\mathbf{x}_{\mathcal{K}}\right)\right)
$$

where upper indices correspond to Newton's iterations. $\mathbf{x}_{\mathcal{K}, \Gamma_{L}}$ is the point which corresponds to $\mathbf{x}_{\mathcal{K}}$ on the left boundary $\Gamma_{L}$ taking into account deformations. In other words, the initial guess of the pressure remains constant along streamlines of the solid deformations.

The initial guess of the saturation satisfies $S_{\mathcal{K}}^{0} \in\left(S_{r}, 1\right)$ for all $\mathcal{K} \in \mathcal{T}$. Thus, the initial guess $\mathcal{T}_{1}^{0}$ is an empty set and the initial guess $\mathcal{T}_{2}^{0}$ is equal to $\mathcal{T}$. After each Newton's iteration $k$, when correction values for pressure $\Delta p_{\mathcal{K}}^{k+1}$ and saturation $\Delta S_{\mathcal{K}}^{k+1}$ are computed, we define $p_{\mathcal{K}}^{k+1}$ as:

$$
p_{\mathcal{K}}^{k+1}=p_{\mathcal{K}}^{k}+\Delta p_{\mathcal{K}}^{k+1} \text { for all } \mathcal{K} \in \mathcal{T}
$$

and the simple restriction operator is applied to define $S_{\mathcal{K}}^{k+1}$ :

$$
S_{\mathcal{K}}^{k+1}= \begin{cases}S_{r}, & \text { if } S_{\mathcal{K}}^{k}+\Delta S_{\mathcal{K}}^{k+1} \leq S_{r} \\ S_{\mathcal{K}}^{k}+\Delta S_{\mathcal{K}}^{k+1}, & \text { if } S_{\mathcal{K}}^{k}+\Delta S_{\mathcal{K}}^{k+1} \in\left(S_{r}, 1\right) \\ 1, & \text { if } S_{\mathcal{K}}^{k}+\Delta S_{\mathcal{K}}^{k+1} \geq 1\end{cases}
$$


for all $\mathcal{K} \in \mathcal{T}$. Then, the sets $\mathcal{T}_{1}^{k+1}$ and $\mathcal{T}_{2}^{k+1}$ are defined as:

$$
\begin{aligned}
& \mathcal{T}_{1}^{k+1}=\left\{\mathcal{K} \in \mathcal{T}: S_{\mathcal{K}}^{k+1}=S_{r} \text { or } S_{\mathcal{K}}^{k+1}=1\right\} \\
& \mathcal{T}_{2}^{k+1}=\left\{\mathcal{K} \in \mathcal{T}: S_{\mathcal{K}}^{k+1} \in\left(S_{r}, 1\right)\right\}
\end{aligned}
$$

Remark 3 The proposed numerical procedure (39)-(42) may cause an appearance of some unphysical domains with the water saturation being equal to $S_{r}$. This domain is required for the completeness of the numerical approach. From a physical point of view, in the domain where this regime appears the following equations have to be satisfied:

$$
p_{\mathcal{K}}=-p_{c}^{\text {stat }}\left(S_{r}\right), \quad S_{\mathcal{K}}=S_{r} .
$$

In practice, we do not observe numerical experiments where single-phase air flow appears.

\section{Numerical Experiments}

This section presents numerical experiments for the pressing section of a paper machine. At first, single-layer test cases are considered to evaluate the behavior of the solution in presence of the dynamic capillary effect and to compare the results with the laboratory experiments presented in Beck (1983). Then, we study how the dynamic capillarity acts in the multilayer case. Since in this work we suggested to use the MPFA-O FV scheme for discretizing the governing equations at the end of this section we compare numerical results with the results earlier obtained in Rief (2005) using the FE scheme with the static capillary pressure.

All tests are performed with realistic sets of parameters. More detailed description of the parameter evaluation can be found in Rief (2007).

4.1 Numerical experiments for the evaluation of the dynamic capillary effect: single-layer case

Simulation results for three different test cases with single layer configuration are presented. Sets of parameters correspond to two types of felts and a paper. For the dynamic capillary pressure model we consider the material coefficient $\tau$ equal to 0 , 10 and $100 \mathrm{Pas}$. The case $\tau=0$ corresponds to the static capillary pressure. Our studies of a one-dimensional model in Iliev et al. (2012) indicated that values of $\tau$ of order 10 and $100 \mathrm{Pas}$ are realistic for the process studied in this paper. Further on, we consider cases with different velocities $\mathbf{V}_{s, \text { in }}$ and with different initial saturation $C_{0}$.

The input data is presented in Tables 1,2. Here we give the input data only for the flow model. For the typical parameters of the elasticity model we refer to Rief (2007). As it was mentioned in Section 2.7, the elasticity model is used to obtain the geometry of the computational domain $\Omega$, the distributions of the porosity $\phi(\mathbf{x})$, and the solid velocity $\mathbf{V}_{s}(\mathbf{x})$. As an example, the typical distributions of these parameters are shown for the first test case "Felt 1" with $\left|\mathbf{V}_{s, i n}\right|=100 \mathrm{~m} / \mathrm{min}$ in Fig. 8, where in Fig. 8A the porosity $\phi$ is presented. In Figs. 8B and $\mathrm{C} x$ and $z$-components of the solid velocity $\mathbf{V}_{s}$ are shown, respectively. 
Table 1 Experimental data for all test cases (Rief, 2007)

\begin{tabular}{ccc}
\hline Variable & Dimension & Value \\
\hline$k_{r}$ & {$[-]$} & $S^{3.5}$ \\
$\mathbf{K}$ & {$\left[\mathrm{m}^{2}\right]$} & $\mathbf{K}_{0} \frac{\phi^{3}}{(1-\phi)^{2}}$ \\
$\mu$ & {$[P a s]$} & 0.0008 \\
$p_{c}^{\text {stat }}$ & {$[P a]$} & $a(\phi-1)\left(\frac{1}{S_{-}-S_{r}}-\frac{1}{1-S_{r}}\right)^{1 / 2}$ \\
$a$ & {$[P a]$} & $\frac{P_{0}}{1-\phi_{0}}\left(\frac{1}{C_{0}-S_{r}}-\frac{1}{1-S_{r}}\right)^{-1 / 2}$ \\
$S_{r}$ & {$[-]$} & 0.1 \\
$P_{0}$ & {$[P a]$} & -5000 \\
\hline
\end{tabular}

Table 2 Experimental data for different fabrics

\begin{tabular}{ccccc}
\hline Variable & Dimension & Felt 1 & Felt 2 & Paper \\
\hline $\mathbf{K}_{0, x x}$ & {$\left[\mathrm{~m}^{2}\right]$} & $2.95 e-11$ & $1.57 e-11$ & $5.00 e-12$ \\
$\mathbf{K}_{0, x y}$ & {$\left[\mathrm{~m}^{2}\right]$} & $-6.66 e-14$ & $-1.43 e-13$ & 0 \\
$\mathbf{K}_{0, y y}$ & {$\left[\mathrm{~m}^{2}\right]$} & $1.82 e-11$ & $2.96 e-11$ & $1.00 e-13$ \\
$\left.\phi\right|_{\Gamma_{L}}$ & {$[-]$} & 0.45 & 0.34 & 0.88 \\
$\left.d\right|_{\Gamma_{L}}$ & {$[\mathrm{~mm}]$} & 0.40 & 0.60 & 0.28 \\
$C_{0}$ & {$[-]$} & $0.25,0.35$ & $0.3,0.5$ & $0.4,0.6$ \\
$\Gamma_{L}$ & {$[\mathrm{~m}]$} & & -0.05 & \\
$\Gamma_{R}$ & {$[\mathrm{~m}]$} & & 0.05 & \\
$\left|\mathbf{V}_{s, i n}\right|$ & {$[\mathrm{m} / \mathrm{min}]$} & & 100,300 & \\
\hline
\end{tabular}

The obtained distributions of the water saturation and the water pressure in the single-layer case show a homogeneous behavior in the vertical direction. Therefore, all numerical results in this subsection are shown as one-dimensional graphs, representing vertical averages of two-dimensional values. Simulation results for "Felt 1", "Felt 2" and "Paper" are shown in Figs. 9, 10, in Figs. 11, 12 and Figs. 13, 14, respectively. Figs. 9, 11, 13 correspond to $\left|\mathbf{V}_{s, \text { in }}\right|=100 \mathrm{~m} / \mathrm{min}$, while Figs. 10, 12, 14 correspond to $\left|\mathbf{V}_{s, i n}\right|=300 \mathrm{~m} / \mathrm{min}$. Figs. 9A-14A illustrate the computed saturation, while in Figs. 9B-14B the computed fluid pressure is shown. Further on, Figs. 9C-14C represent different magnification of part of the data, aiming at better visualization. These figures represent only part of the results, namely those which can not be well seen in Figs. 9B-14B. For every test case we vary the initial saturation to see the influence of the dynamic capillary pressure model in case of the unsaturated and saturated water flow. For "Felt 1 " we consider two values of $C_{0}$, which are 0.25 and 0.35 , for "Felt 2 " the initial saturation is equal to 0.3 and 0.5 , and for "Paper" $C_{0}$ is equal to 0.4 and 0.6 . In Figs. 9-14 the data which corresponds to the same initial saturation is shown with the same type of markers. The data corresponding to the same value of $\tau$ we present with the same color.

In general, we see that the two-dimensional model in the single-layer case shows the same kind of behavior of the pressure and the saturation in presence of the dynamic capillary effect as the one-dimensional model considered in Iliev et al. (2012). With the increase of the material coefficient $\tau$ we observe a decrease of the maximum value of the saturation or a reduction of the fully saturated zone. Regarding the distribution of the pressure, with the increase of $\tau$ the maximum value of the pressure decreases a little bit in case when saturated flow is present and it sifts to 
the left in case of the unsaturated flow. For both flow regimes we observe a decrease of the pressure below the initial value behind the center of the pressing zone. These effects are seen better for the fabrics "Felt 1" and "Felt 2". For the "Paper" fabric we obtain similar but less evident behavior. This kind of the water pressure behavior was also observed in the laboratory experiment by Beck (1983).

In Fig. 15A the dependence of the fluid pressure peak on the initial saturation is shown for all test cases with different material coefficients $\tau$ and fixed $\left|\mathbf{V}_{s, i n}\right|=$ $100 \mathrm{~m} / \mathrm{min}$. This numerical experiment shows that for small initial saturation the dynamic capillary pressure model significantly influences the fluid pressure peak. But when the initial saturation becomes larger, the pressure peak increases and does not differ much for the static and dynamic capillary pressure models. We also observe that the values of $C_{0}$ after which pressure peak increases depends on the test case.

For better understanding of the behavior of the fluid pressure let us introduce the following quantity $Q_{i n}$ :

$$
Q_{\text {in }}=C_{0} \frac{\phi\left(x_{L}\right) d\left(x_{L}\right)}{\phi\left(x_{*}\right) d\left(x_{*}\right)},
$$

where $d$ is the one-dimensional function of the $x$-coordinate which expresses the thickness of the layer, $x_{L}$ is the $x$-coordinate of the left boundary $\Gamma_{L}, x_{*}$ is the $x$ coordinate where the layer reaches the minimum thickness or the maximum value of the porosity during pressing. In other words, the quantity $Q_{i n}$ expresses the ratio of incoming water volume to void volume at the center of the nip. In Fig. 15B we show the dependence of the fluid pressure peak on $Q_{i n}$. When $Q_{i n}$ become greater than one, a fully saturated zone appears and the fluid pressure rises dramatically. In Beck (1983) a similar dependence is presented. They observe the same behavior of the fluid pressure for $Q_{i n}<1.3$. But when $Q_{i n}$ exceeds 1.3, the pressure reaches a metastable state and does not increase much with increase of the initial saturation due to the water escape through the entrance of the nip. In our model water rearranges within the computational domain but it is not allowed to escape from the computational domain. So we do not observe this stabilization of the fluid pressure peak due to the model limitations. Enrichment of the model with the boundary conditions which allow escape of the water through the upper and lower boundaries is planned as the next step of our future studies.

\subsection{Numerical investigation of the dynamic capillary effect: multilayer case}

Now we consider the multilayer cases which may be investigated numerically only with the help of the two-dimensional model. The input data from Table 1 is used in all numerical experiments.

The first test case is developed for the roll press with eleven layers (see Table 3), where Layer 6 presents the paper. The paper-felt sandwich is transported with the speed $\left|\mathbf{V}_{s, i n}\right|=100 \mathrm{~m} / \mathrm{min}$. The boundaries of the computational domain are considered to be $\Gamma_{L}=\{x=-0.1 m\}, \Gamma_{R}=\{x=0.1 m\}$. Remembering that $\tau$ equal to zero corresponds to the static capillary pressure model we show the numerical results for the first test case in Figs. 16-19. Figs. 16A, B, C show the distribution of the water saturation for $\tau$ equal to 0,10 , and $100 \mathrm{~Pa}$ s, respectively. In Figs. 17A, B, $\mathrm{C}$ the location of the fully saturated zone and in Figs. 18A, B, C the distribution of the fluid pressure are shown for $\tau$ equal to 0, 10, and $100 \mathrm{~Pa}$ s, respectively. Fig. 19 

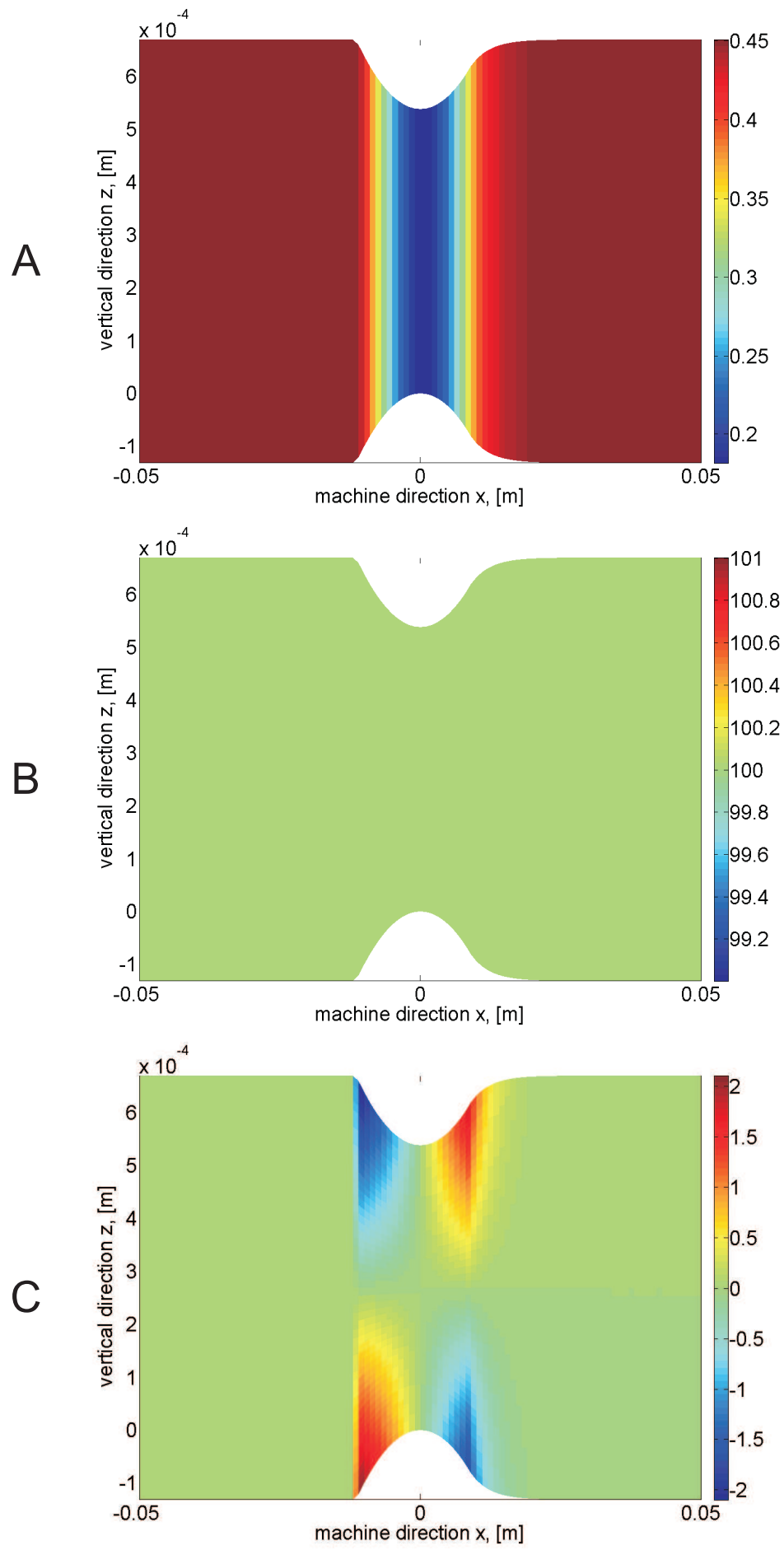

Fig. 8 Input data for the flow solver for the first test case "Felt 1" with $\left|\mathbf{V}_{s, \text { in }}\right|=100 \mathrm{~m} / \mathrm{min}$ : $\mathrm{A}$ - the porosity $\phi, \mathrm{B}-x$-component of the solid velocity $\mathbf{V}_{s}, \mathrm{C}-z$-component of the solid velocity $\mathbf{V}_{s}$ 

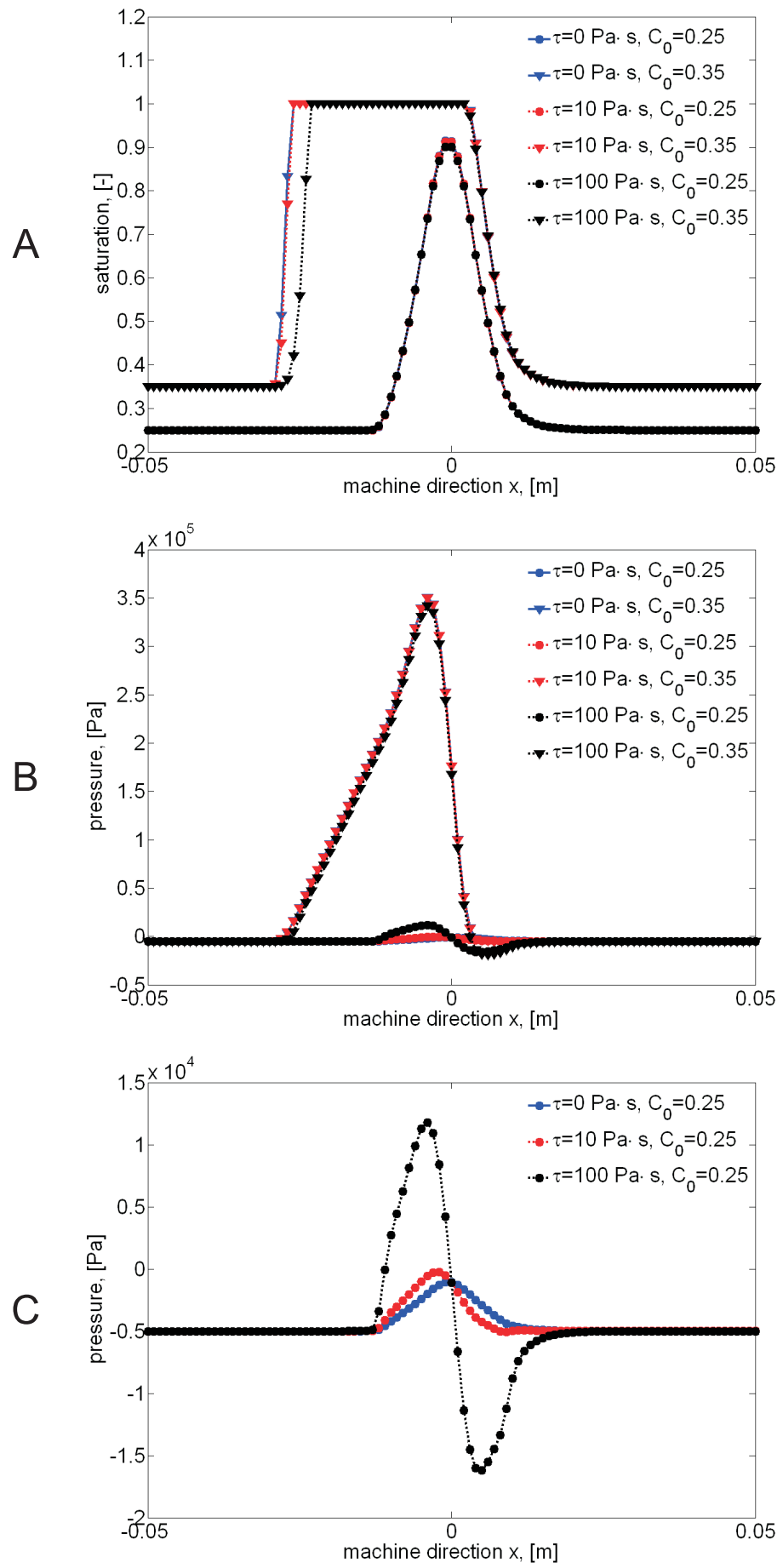

Fig. 9 Saturation (A) and pressure (B, C) for "Felt 1" with $\left|\mathbf{V}_{s, i n}\right|=100 \mathrm{~m} / \mathrm{min}$ 

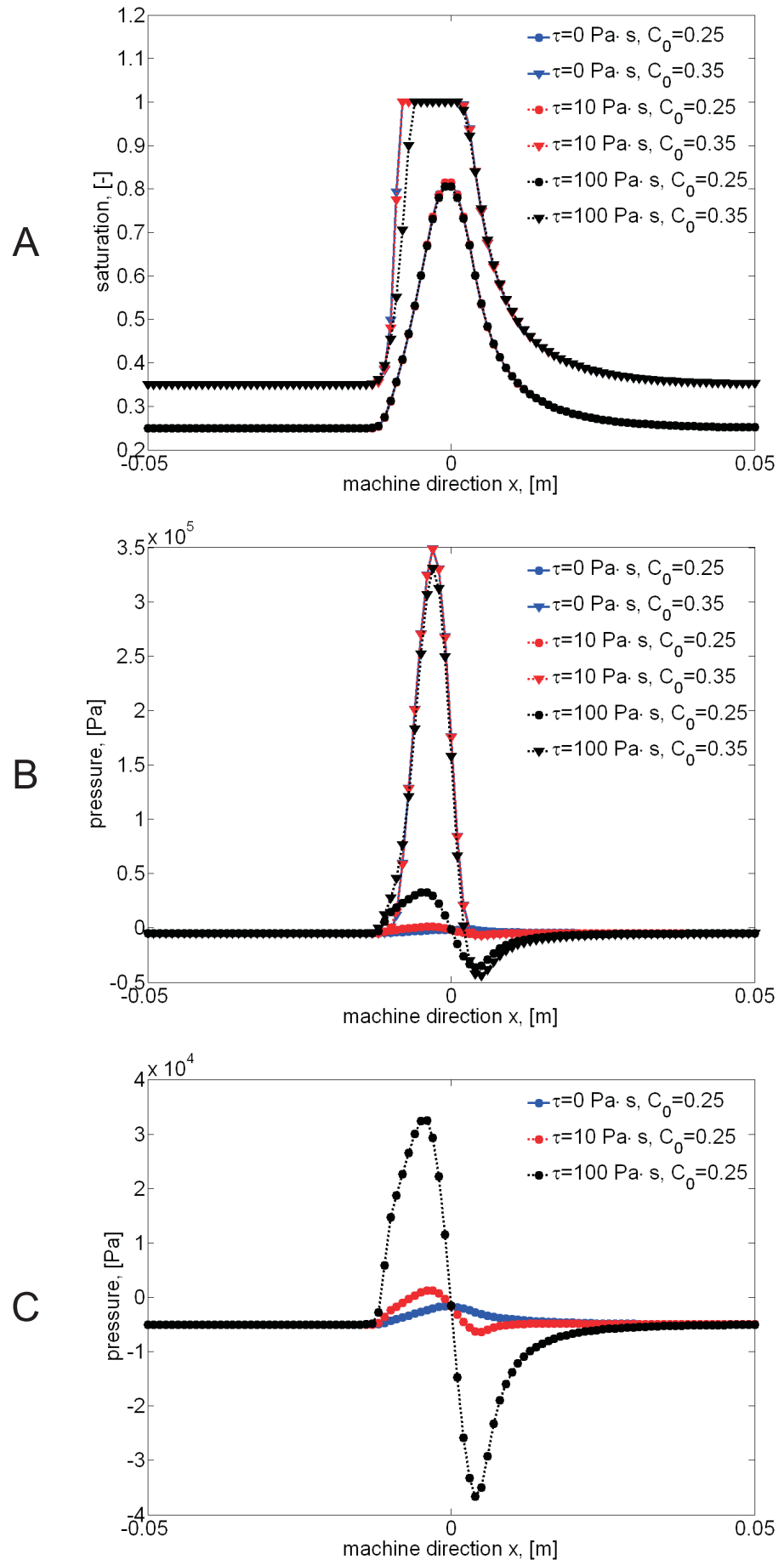

Fig. 10 Saturation (A) and pressure (B, C) for "Felt 1" with $\left|\mathbf{V}_{s, i n}\right|=300 \mathrm{~m} / \mathrm{min}$ 

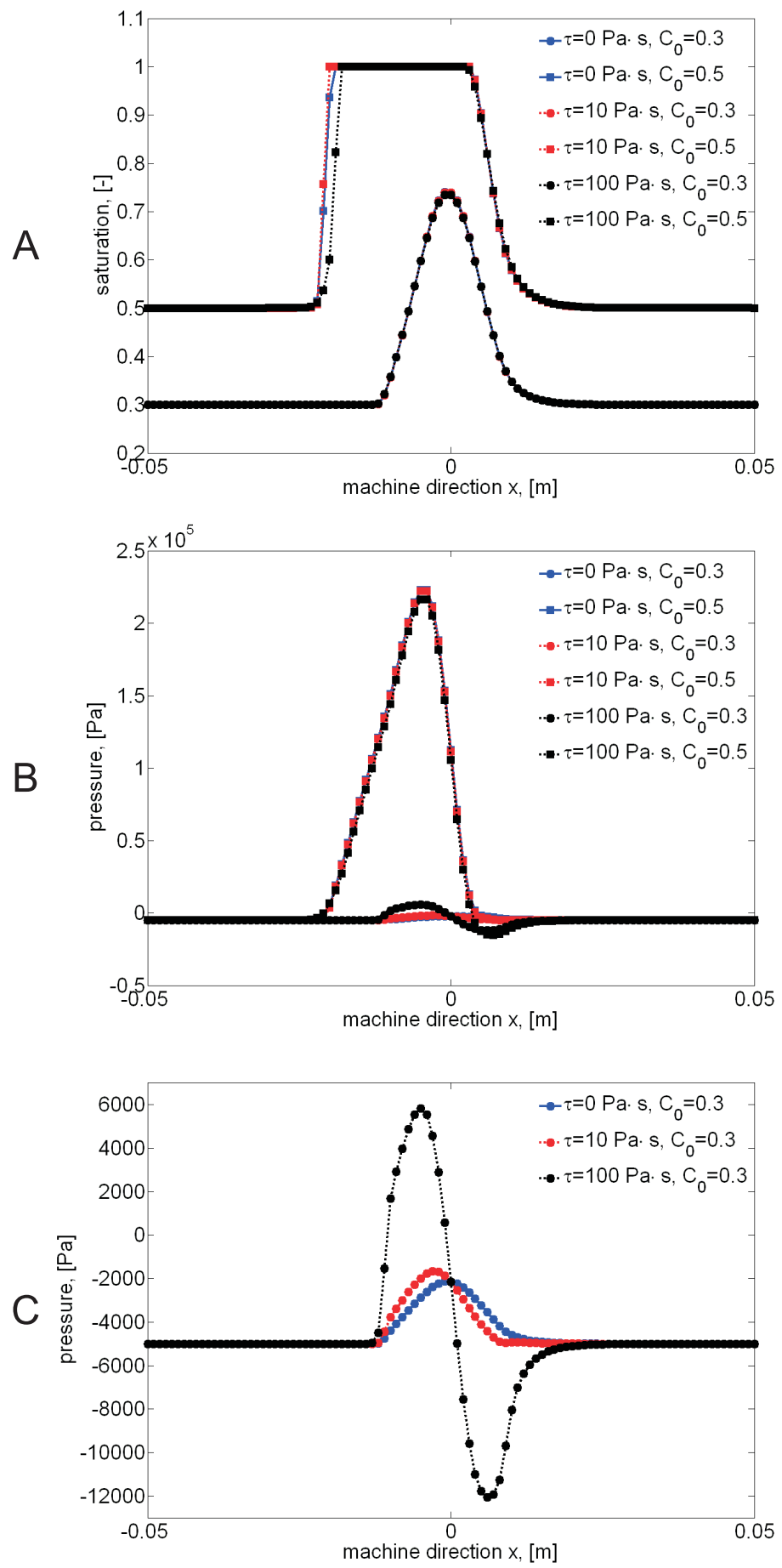

Fig. 11 Saturation (A) and pressure (B, C) for "Felt 2" with $\left|\mathbf{V}_{s, i n}\right|=100 \mathrm{~m} / \mathrm{min}$ 

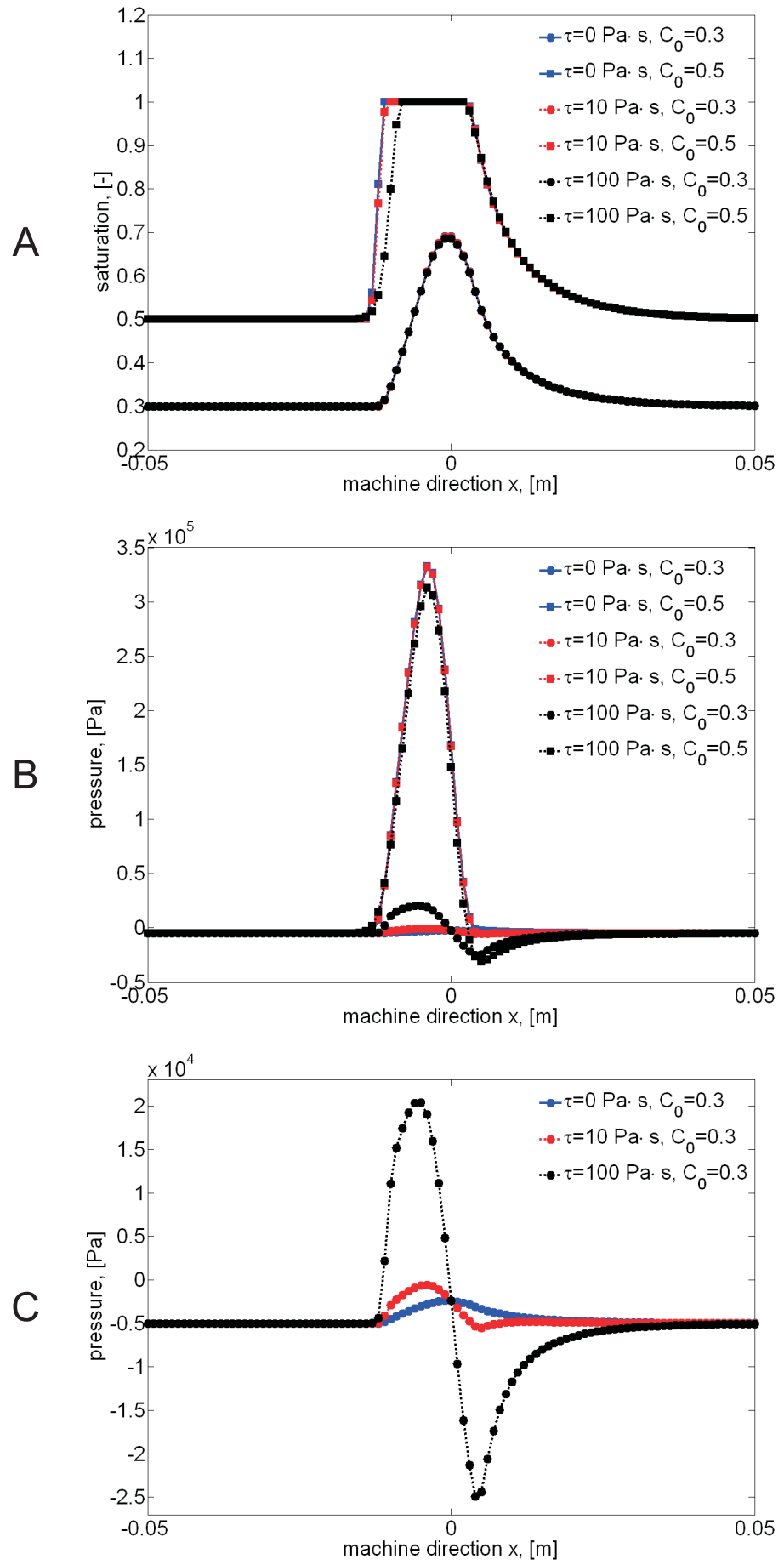

Fig. 12 Saturation (A) and pressure (B, C) for "Felt 2" with $\left|\mathbf{V}_{s, i n}\right|=300 \mathrm{~m} / \mathrm{min}$ 

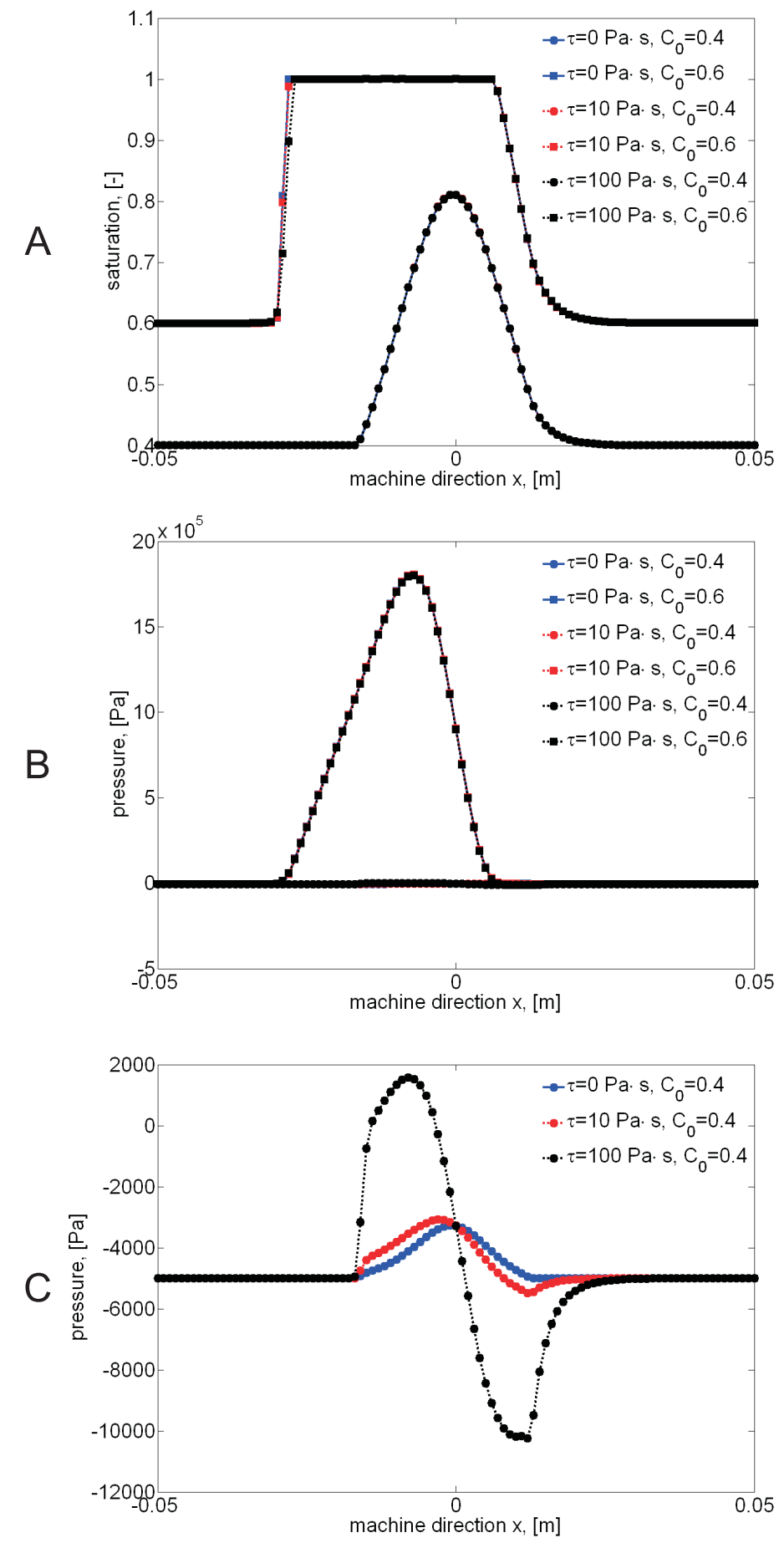

Fig. 13 Saturation (A) and pressure (B, C) for "Paper" with $\left|\mathbf{V}_{s, i n}\right|=100 \mathrm{~m} / \mathrm{min}$ 

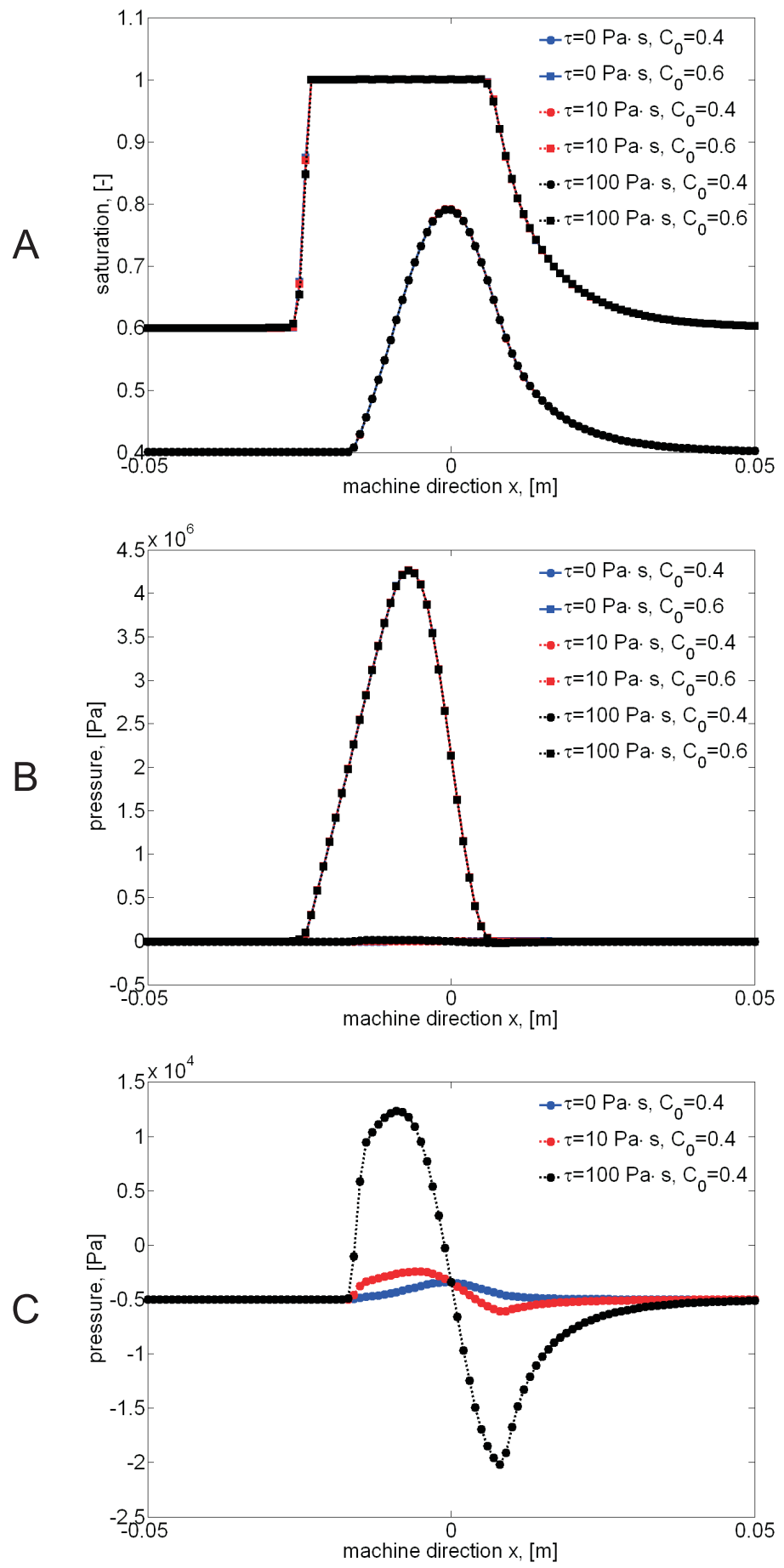

Fig. 14 Saturation (A) and pressure (B, C) for "Paper" with $\left|\mathbf{V}_{s, i n}\right|=300 \mathrm{~m} / \mathrm{min}$ 

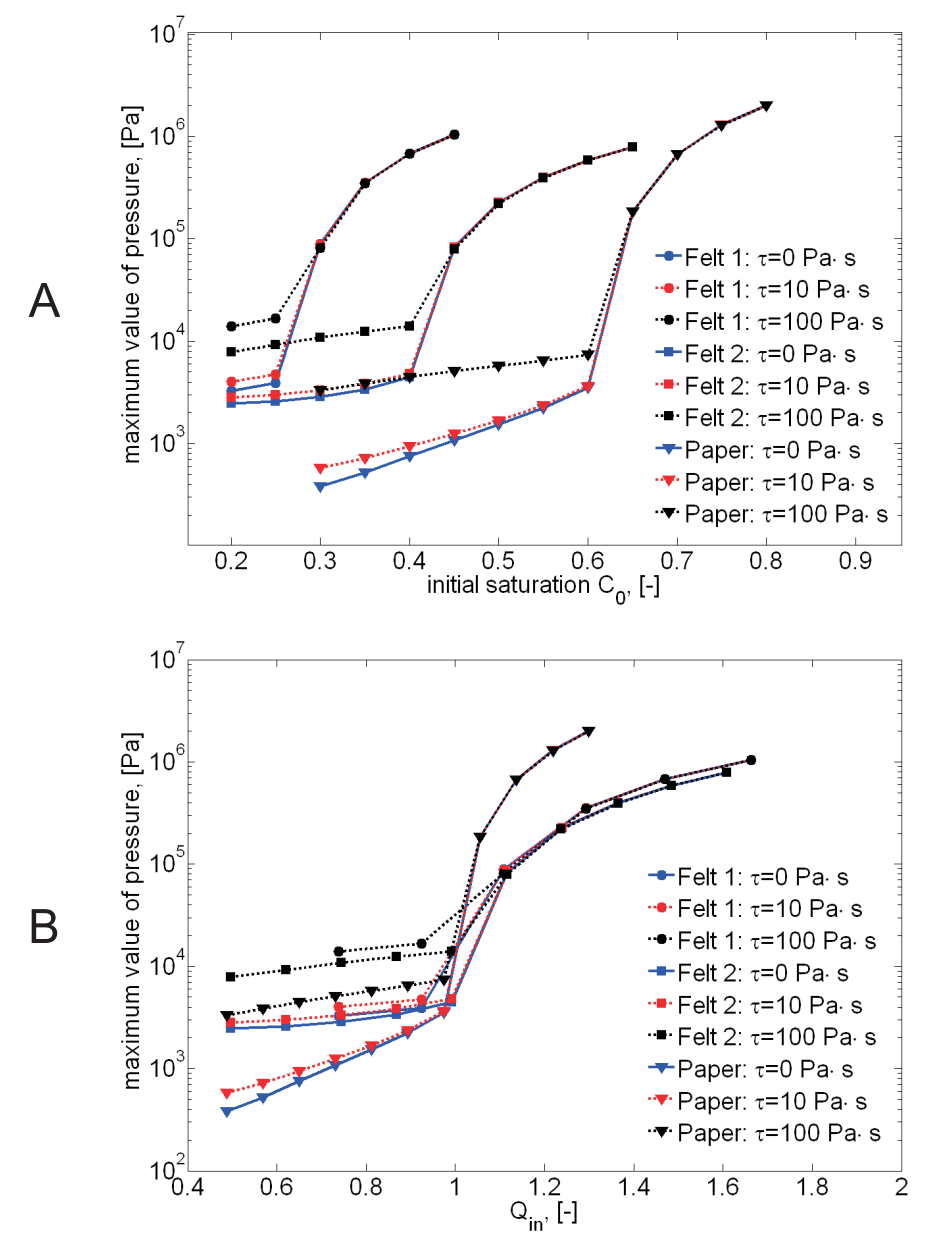

Fig. 15 Fluid pressure peak as a function of initial saturation (A) and $Q_{i n}$ (B) for $\left|\mathbf{V}_{s, i n}\right|=$ $100 \mathrm{~m} / \mathrm{min}$

presents the dry solid content of the paper layer for the different values of $\tau$. As we can see from the obtained numerical results, the behavior of the solution to the multilayer test problem is quite similar to the single-layer tests. The fully saturated zone decreases and the fluid pressure takes the characteristic shape with increase of the material coefficient $\tau$. We also notice that the dry solid content of the paper is not influenced much by the dynamic capillary effect. It changes the shape with the increase of $\tau$ but the final value remains the same.

The second numerical test is developed for the roll press with parameters presented in Table 4 and $\left|\mathbf{V}_{s, \text { in }}\right|=500 \mathrm{~m} / \mathrm{min}$. The boundaries of the computational domain are $\Gamma_{L}=\{x=-0.15 m\}, \Gamma_{R}=\{x=0.15 m\}$. The numerical results are presented in Figs. 20-23. The saturation for $\tau$ equal to 0, 10, and $100 \mathrm{~Pa} s$ is shown in Figs. 20A, B, and C, respectively. The location of the fully saturated zone and the distribution of pressure are presented in Figs. 21A, B, C and 22A, B, C for the 
Table 3 Experimental data for test case 1

\begin{tabular}{ccccccc}
\hline & $\mathbf{K}_{0, x x},\left[m^{2}\right]$ & $\mathbf{K}_{0, x y},\left[m^{2}\right]$ & $\mathbf{K}_{0, y y},\left[m^{2}\right]$ & $\left.\phi\right|_{\Gamma_{L}},[-]$ & $\left.d\right|_{\Gamma_{L}},[m m]$ & $C_{0},[-]$ \\
\hline Layer 1 & $1.00 e-09$ & 0 & $1.00 e-09$ & 0.20 & 2.50 & 0.26 \\
Layer 2 & $1.89 e-11$ & $-1.89 e-13$ & $5.91 e-11$ & 0.40 & 0.28 & 0.38 \\
Layer 3 & $1.57 e-11$ & $-1.43 e-13$ & $2.96 e-11$ & 0.34 & 0.60 & 0.44 \\
Layer 4 & $6.72 e-12$ & $-6.51 e-14$ & $2.42 e-11$ & 0.31 & 0.52 & 0.45 \\
Layer 5 & $8.34 e-11$ & $-1.05 e-13$ & $2.46 e-11$ & 0.52 & 0.60 & 0.42 \\
Layer 6 & $5.00 e-12$ & 0 & $1.00 e-13$ & 0.88 & 0.28 & 0.90 \\
Layer 7 & $2.95 e-11$ & $-6.66 e-14$ & $1.82 e-11$ & 0.45 & 0.40 & 0.44 \\
Layer 8 & $2.93 e-12$ & $-5.22 e-14$ & $1.59 e-11$ & 0.25 & 0.42 & 0.45 \\
Layer 9 & $8.36 e-12$ & $-8.88 e-14$ & $1.36 e-11$ & 0.29 & 0.65 & 0.44 \\
Layer 10 & $1.11 e-11$ & $-1.13 e-13$ & $3.02 e-11$ & 0.31 & 0.28 & 0.48 \\
Layer 11 & $8.17 e-11$ & $-1.05 e-13$ & $6.48 e-11$ & 0.53 & 0.23 & 0.49 \\
\hline
\end{tabular}

Table 4 Experimental data for test case 2

\begin{tabular}{ccccccc}
\hline & $\mathbf{K}_{0, x x},\left[m^{2}\right]$ & $\mathbf{K}_{0, x y},\left[m^{2}\right]$ & $\mathbf{K}_{0, y y},\left[m^{2}\right]$ & $\left.\phi\right|_{\Gamma_{L}},[-]$ & $\left.d\right|_{\Gamma_{L}},[m m]$ & $C_{0},[-]$ \\
\hline Layer 1 & $5.00 e-12$ & 0 & $1.00 e-13$ & 0.88 & 0.24 & 0.91 \\
Layer 2 & $1.51 e-10$ & $1.64 e-12$ & $1.15 e-10$ & 0.53 & 0.51 & 0.51 \\
Layer 3 & $1.45 e-10$ & $2.34 e-12$ & $1.60 e-10$ & 0.53 & 0.81 & 0.51 \\
Layer 4 & $3.46 e-10$ & $-5.60 e-13$ & $2.05 e-10$ & 0.57 & 2.65 & 0.51 \\
Layer 5 & $9.75 e-10$ & $-2.88 e-12$ & $4.93 e-10$ & 0.80 & 0.65 & 0.51 \\
Layer 6 & $1.00 e-08$ & 0 & $1.00 e-08$ & 0.35 & 5.00 & 0.17 \\
\hline
\end{tabular}

different values of the material coefficient, respectively. Here we observe a significant decrease of the fully saturated zone with increase of the dynamic component. The fluid pressure shows the same behavior as before. After the peak of the pressure, we observe with increase of $\tau$ an appearance of the region with the pressure below the initial value. As opposed to the previous example, the dry solid content of the paper is influenced by the dynamic capillarity. Its value increases a little bit after the pressing with increasing $\tau$.

For the third numerical test we consider the shoe press with $\left|\mathbf{V}_{s, \text { in }}\right|=1000 \mathrm{~m} / \mathrm{min}$ and $\Gamma_{L}=\{x=-0.30 m\}, \Gamma_{R}=\{x=0.40 m\}$. We use the input data for the layers as in test case 1 from Table 3 except the initial saturation which is presented in Table 5. Numerical results are presented in Figs. 24-27. The difference in the water saturation for the considered values of $\tau$ can not be seen. Thus, we show only one distribution of the water saturation in Fig. 24, where Figs. 24(A) and (B) show the water saturation in the undeformed and standard computational domains, respectively. Figs. 25A, 26A correspond to the static capillary pressure model. In Figs. 25B, 26B and Figs. 25C, $26 \mathrm{C}$ the material coefficient $\tau$ is equal to 10 and $100 \mathrm{Pas}$, respectively. The location of the fully saturated zone are shown in Fig. 25. Fig. 26 represents the distribution of the fluid pressure. The dry solid content of the paper layer is shown in Fig. 27 for different $\tau$. All numerical results are presented for the undeformed geometry except the saturation for $\tau=100 \mathrm{~Pa}$. The fluid pressure shows the same behavior as in the previous test cases. But in saturation we observe an increase of the fully saturated zone with increasing $\tau$. It may be caused by the different geometries of the computational domain. The curve of the dry solid content changes its shape but the final value remains the same for the cases with the dynamic and static capillary pressure. 
Table 5 Experimental data for test case 3

\begin{tabular}{cc}
\hline & $C_{0},[-]$ \\
\hline Layer 1 & 0.12 \\
Layer 2 & 0.38 \\
Layer 3 & 0.44 \\
Layer 4 & 0.45 \\
Layer 5 & 0.42 \\
Layer 6 & 0.99 \\
Layer 7 & 0.44 \\
Layer 8 & 0.45 \\
Layer 9 & 0.44 \\
Layer 10 & 0.48 \\
Layer 11 & 0.49 \\
\hline
\end{tabular}

\subsection{Numerical investigation of the discretization technique}

For the model with the static capillary pressure we have the possibility to compare the numerical solution with results obtained in Rief (2005), where the model was discretized with the finite element method. This opportunity is used to investigate the quality of the discretization technique used in this study. Typically, the difference in solutions can be well seen in the distribution of the water velocity. For the first and third test cases we show distributions of the water velocities in Figs. 28, 29. In these figures we do not show the whole range of the water velocity in order to see better regions with nonphysical values. We cut the water velocities by some value which is shown in each figure on the color bar (see Figs.28, 29). Figs. 28A and 29A represent the distribution of the water velocity obtained with the help of our model. The results obtained with the help of the model proposed by Rief are shown in Figs. 28B and 29B. In Figs. 28C, 29C we show magnified regions which are indicated in Figs. 28B, 29B with the help of black boxes. The last figures show that the solution obtained with the help of discretization used by Rief gives nonsmooth and sometimes oscillatory solution at the same time as our solution is smooth. Such nonphysical oscillations of the finite element solution are typical for convection-diffusion equations, if no stabilization technique (e.g. streamwise diffusion) is used.

In most of the test cases it was observed that the numerical algorithm proposed in this study converges faster than the algorithm from Rief (2005). The MPFA-O method is also very well applicable to the specific boundary conditions which we have to preserve between layers.

\section{Conclusions}

In this work a two-dimensional model was developed for the pressing section of a paper machine. This model adopts the dynamic capillary effects described earlier by Hassanizadeh and Gray. At first, the mathematical model was discussed together with its discretization technique. Then, some numerical results were obtained. Singlelayer test cases were carried out to compare the two-dimensional solutions with the laboratory experiments and to obtain the main behavior of the water saturation and the water pressure in presence of the dynamic capillary effects. The behavior of the pressure for the model with the dynamic capillary pressure is similar to the behavior 
of the pressure obtained in the laboratory experiments by Beck (1983). We also observed the same kind of dependence of the pressure peak on the initial saturation as Beck.

Multilayer simulations showed that the behavior of the fluid pressure is the same as in the single-layer case. Regarding the distribution of the saturation, we notice that the behavior of the fully saturated regions for the static and dynamic capillary pressure models may differ for different geometries of the computational domain. So we observed a decrease of the fully saturated area with increasing $\tau$ for the roll nips and otherwise for the shoe press. For the dry solid content of the paper layer it was not possible to evaluate a general behavior for all test cases. We observed dependence of the dry solid content on particular test cases. In general, the numerical experiments showed that the material coefficient $\tau$ of order 10 and 100 Pas significantly influences the distributions of the fluid pressure and the saturation. On the other hand the distribution of the dry solid content of the paper layer does not change much when $\tau$ changes in this range.

Acknowledgements The authors would like to thank our industrial partner, Voith Paper Fabric and Roll Systems GmbH at Heidenheim, for the interesting discussions and for the experimental data which allowed us to perform the experiments with the realistic sets of parameters.

\section{References}

Aavatsmark, I.: An introduction to multipoint flux approximations for quadrilateral grids. Comput. Geosci. 6: 405-432 (2002)

Aavatsmark, I.: Multipoint flux approximation methods for quadrilateral grids. 9th International Forum on Reservoir Simulation, Abu Dhabi (2007)

Barenblatt, G.I., Gilman, A.A.: Nonequilibrium counterflow capillary impregnation. J. of Eng. Phys., 52:335-339 (1987)

Barenblatt, G.I., Patzek, T.W., Silin, D.B.: The Mathematical Model of NonEquilibrium Effects in Water-Oil displacement. In SPE/DOE 13th Symposium on improved oil recovery, volume SPE 75169, Tusla, USA (2002)

Bear, J.: Dynamics of fluids in porous media. American Elsevier Pub. Co. (1972)

Bear, J., Bachmat, Y.: Introduction to modeling of transport phenomena in porous media. Kluwer, Dordrecht (1990)

Bear, J., Verruijt, A.: Modeling groundwater flow and pollution. Reidel, Dordrecht, the Netherlands (1987)

Beck, D.: Fluid pressure in a press nip: measurements and conclusions. Engineering Conference Proceedings, TAPPI, Atlanta, GA, 475-487 (1983)

Bermond, C.: Establishing the scientific base for energy efficiency in emerging pressing and drying technologies. Applied Thermal Engineering 17(8-10):901-910 (1997)

Bezanovic, D., van Duijn, C.J., Kaasschieter, E.F.: Analysis of paper pressing: the saturated one-dimensional case. J. Appl. Math. Mech. 86(1):18-36 (2006)

Bezanovic, D., van Duijn, C.J., Kaasschieter, E.F.: Analysis of wet pressing of paper: the three-phase model. Part 1: constant air density. Report CASA 05-16 of the Department of Mathematics and Computer Science, Eindhoven, University of Technology (2007) 
Bezanovic, D., van Duijn, C.J., Kaasschieter, E.F.: Analysis of wet pressing of paper: the three-phase model. Part 2: compressible air case. Transp. Porous Med. 67:171187 (2007)

Bourgeat, A., Panfilov, M.: Effective two-phase flow through highly heterogeneous porous media: Capillary nonequilibrium effects. Computational Geosciences, 2:191-215 (1998)

Broocks, R.H., Corey, A.T.: Hydraulic Properties of Porous Media. In Hydrol. Pap., volume 3, Fort Collins, Colorado State University (1964)

Deuflhard, P.: Newton Methods for Nonlinear Problems. Affine invariance and adaptive algorithms. Computational Mathematics, Springer (2004)

Edwards, M.G.: Unstructured, control-volume distributed, full-tensor finite-volume schemes with flow based grids. Comput. Geosci. 2:433-452 (2002)

Eigestad, G.T. and Klausen, R.A.: On the Convergence of the Multi-Point Flux Approximation O-method: Numerical Experiments for Discontinuous Permeability. Wiley Interscience (2005)

Eymard, R., Gallouet, T., Herbin, R.: Finite Volume Methods. An update of the preprint n0 97-19 du LATP, UMR 6632, Marseille, September 1997 (2006)

Hassanizadeh, S.M., Celia, M.A., Dahle, H.K.: Dynamic effect in the capillary pressure-saturation relationship and its impacts on unsaturated flow. Vadose Zone Journal 1:38-57 (2002)

Hassanizadeh, S.M., Gray, W.G.: Mechanics and thermodynamics of multiphase flow in porous media including interphase boundaries. Adv. Water Resour. 13:169-186 (1990)

Hassanizadeh, S.M., Gray, W.G.: Thermodynamic Basis of Capillary Pressure in Porous Media. Water Resour. Res. 29:3389-3405 (1993a)

Helmig, R.: Multiphase flow and Transport Processes in the Subsurface. Springer, (Environmental Engineering), Berlin, Heidelberg (1997)

Herbin, R. and Hubert, F.: Benchmark on discretization schemes for anisotropic diffusion problems on general grids for anisotropic heterogeneous diffusion problems, in Finite Volumes for Complex Applications V, R. Eymard and J.-M. Hérard, eds., Wiley, Hoboken, NJ, 2008, pp. 659-692.

Hiltunen, K.: Mathematical and Numerical Modelling of Consolidation Processes in Paper Machines. PhD Thesis, University of Jyväskylä, Finland (1995)

Iliev, O., Printsypar, G., Rief, S.: On Mathematical Modeling and Simulation of the Pressing Section of a Paper Machine Including Dynamic Capillary Effects: One-Dimensional Model. Transp. Porous Med. 92(1):41-59, DOI:10.1007/s11242011-9890-y, (2012)

Jewett, K., Ceckler, W., Busker, L., and Co, A.: Computer model of a transversal flow nip. AIChE Symposium Series 76:59-70 (200), New York (1980)

Kalaydjian, F.: Dynamic capillary pressure curve for water/oil displacement in porous media: Theory vs. experiment. Society of Petroleum Engineers, SPE 24813:491-506 (1992)

Kataja, M., Hiltunen, K., Timonen, J.: Flow of water and air in a compressible porous medium. A model of wet pressing of paper. J. Phys. D: Appl. Phys. 25: 1053-1063 (1992)

Kelley, C.T.: Iterative Methods for Linear and Nonlinear Equations, Fundamental Algorithms for Numerical Calculations, SIAM, Philadelphia (1995)

Leverett, M.C.: Capillary Behavior in Porous Solids. Transactions of the AIME, 142:152-169 (1941) 
Manthey, S.: Two-phase flow processes with dynamic effects in porous media - parameter estimation and simulation. Dissertation, Institute of Hydraulic Engineering of Stuttgart, Germany (2006)

Metso Corporation: URL: http://www.metso.com/pulpandpaper (May 2010)

Paper academy: URL: http://www.paperacademy.net/855/paper-papermakingmanufacturing/paper-machine-press-section/ (2011)

Rief, S.: Modeling and simulation of the pressing section of a paper machine. Berichte des Fraunhofer ITWM, Nr. 113 (2007)

Rief, S.: Nonlinear Flow in Porous Media. Dissertation, University of Kaiserslautern, Germany (2005)

Ross, P.J., Smettem, K.R.J.: A Simple Treatment of Physical Nonequilibrium Water Flow in Soils. Soil Sci. Soc. Am. J. 64, 1926-1930 (2000)

Van Genuchten, M.T.: A Closed-Form Equation for Predicting the Hydraulic Conductivity of Unsaturated Soils. Soil Sci. Soc. Am. J., 44:892-898 (1980)

Velten, K., Best, W.: Rolling of unsaturated porous materials: Evolution of a fully saturated zone. Physical Review E 62:3891-3899 (2000) 

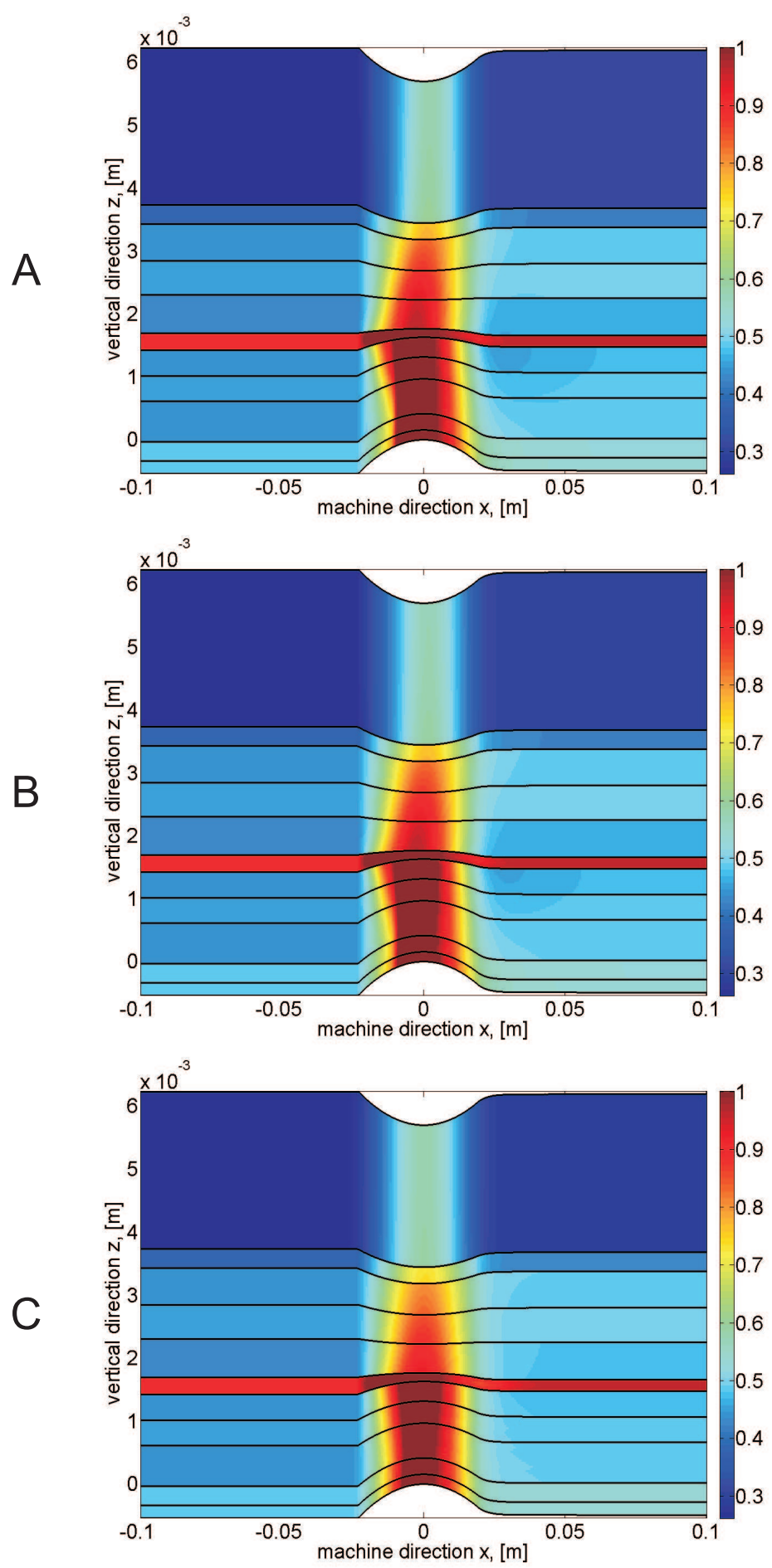

Fig. 16 Saturation for the test case 1 with $\tau$ equal to $0(\mathrm{~A}), 10(\mathrm{~B})$ and $100 \mathrm{Pas}(\mathrm{C})$ 

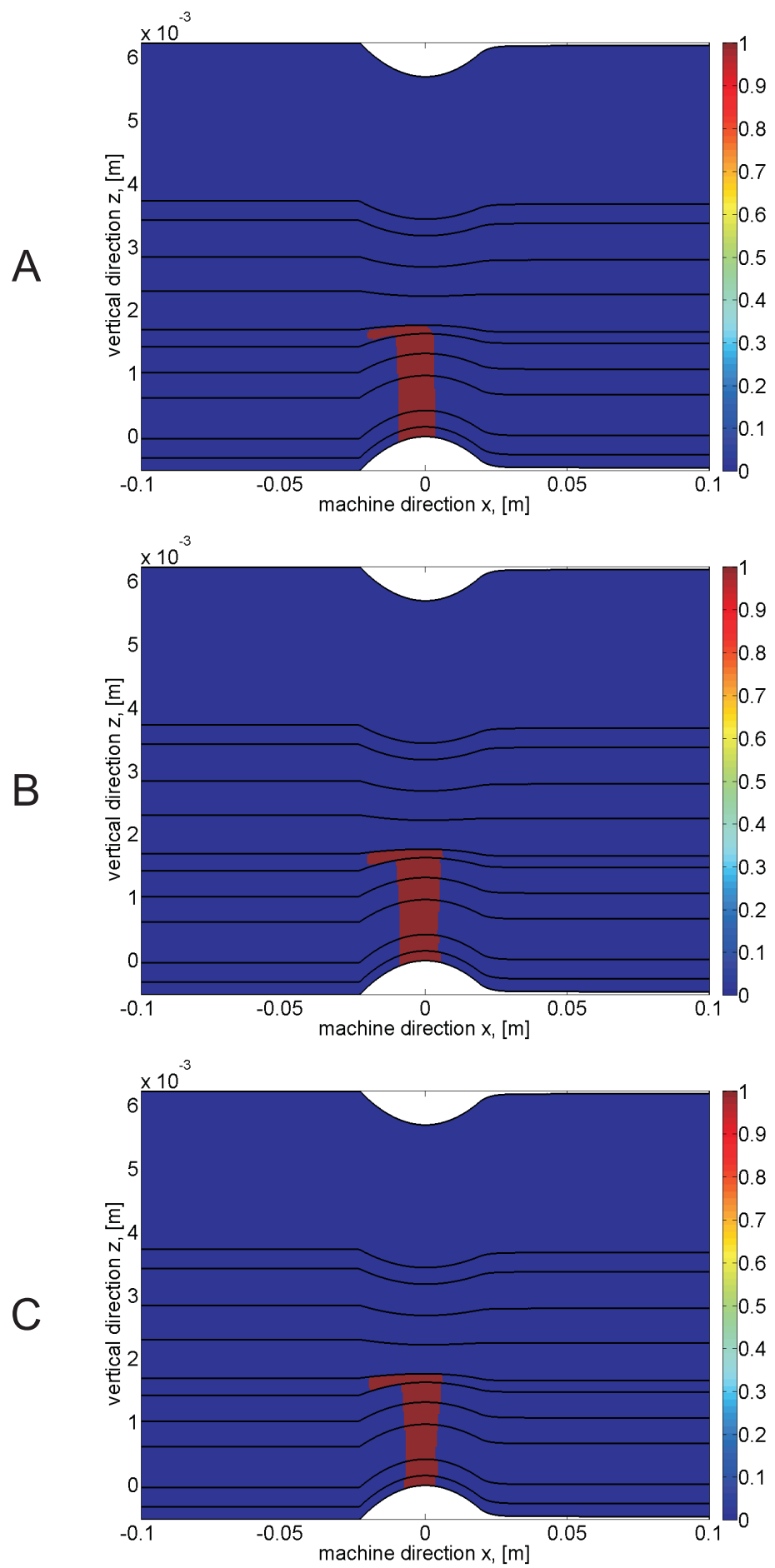

Fig. 17 Fully saturated zone for the test case 1 with $\tau$ equal to 0 (A), 10 (B) and $100 \mathrm{Pas}$ (C) 

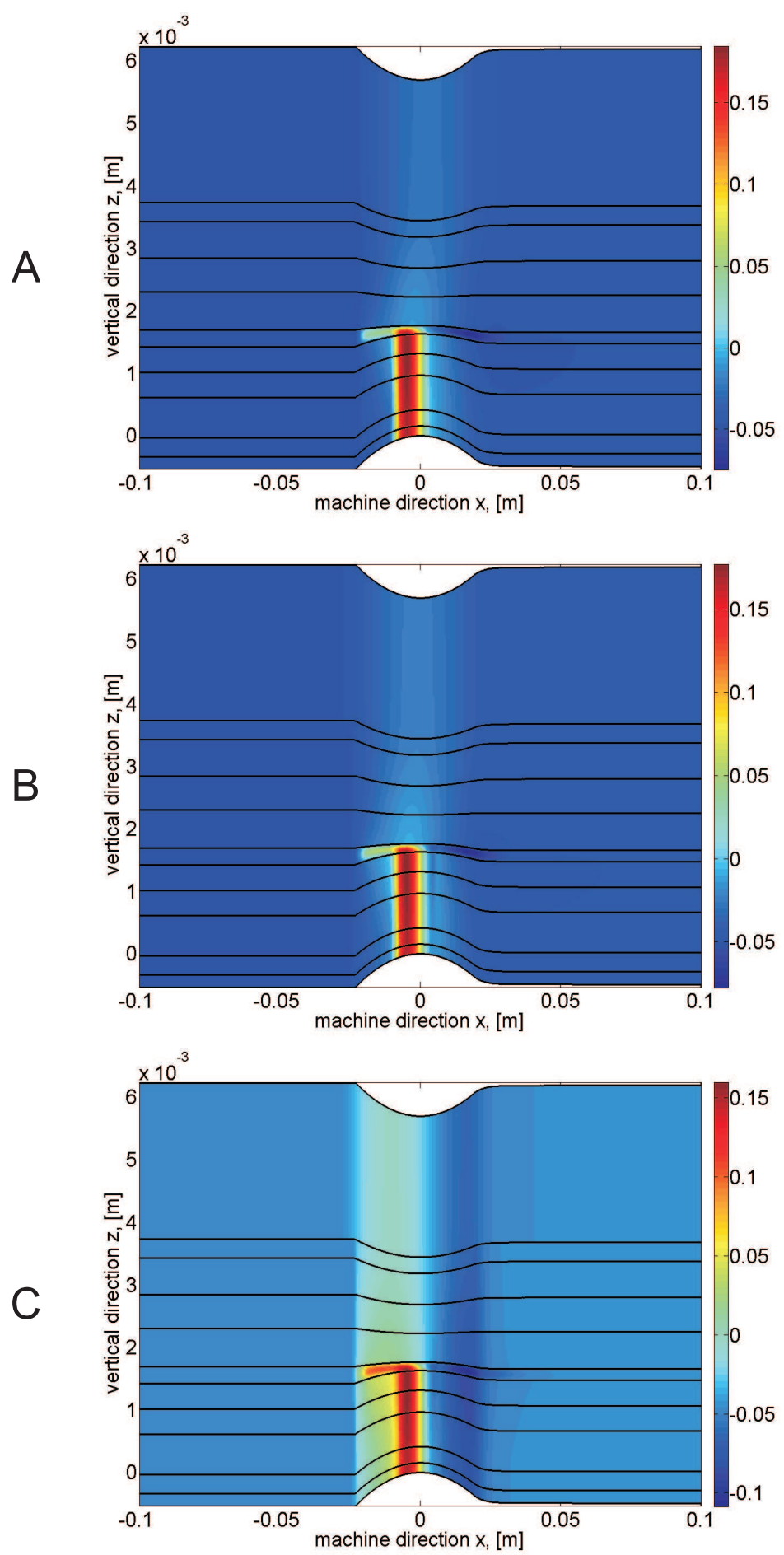

Fig. 18 Pressure for the test case 1 with $\tau$ equal to $0(\mathrm{~A}), 10(\mathrm{~B})$ and $100 \mathrm{~Pa} s(\mathrm{C})$ 


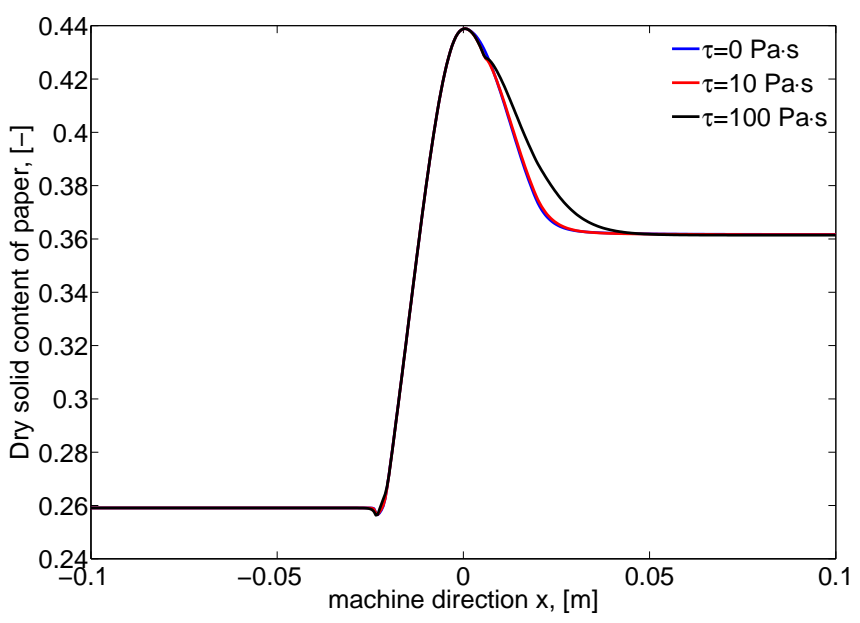

Fig. 19 Dry solid content of the paper for the test case 1 for different values of $\tau$ 

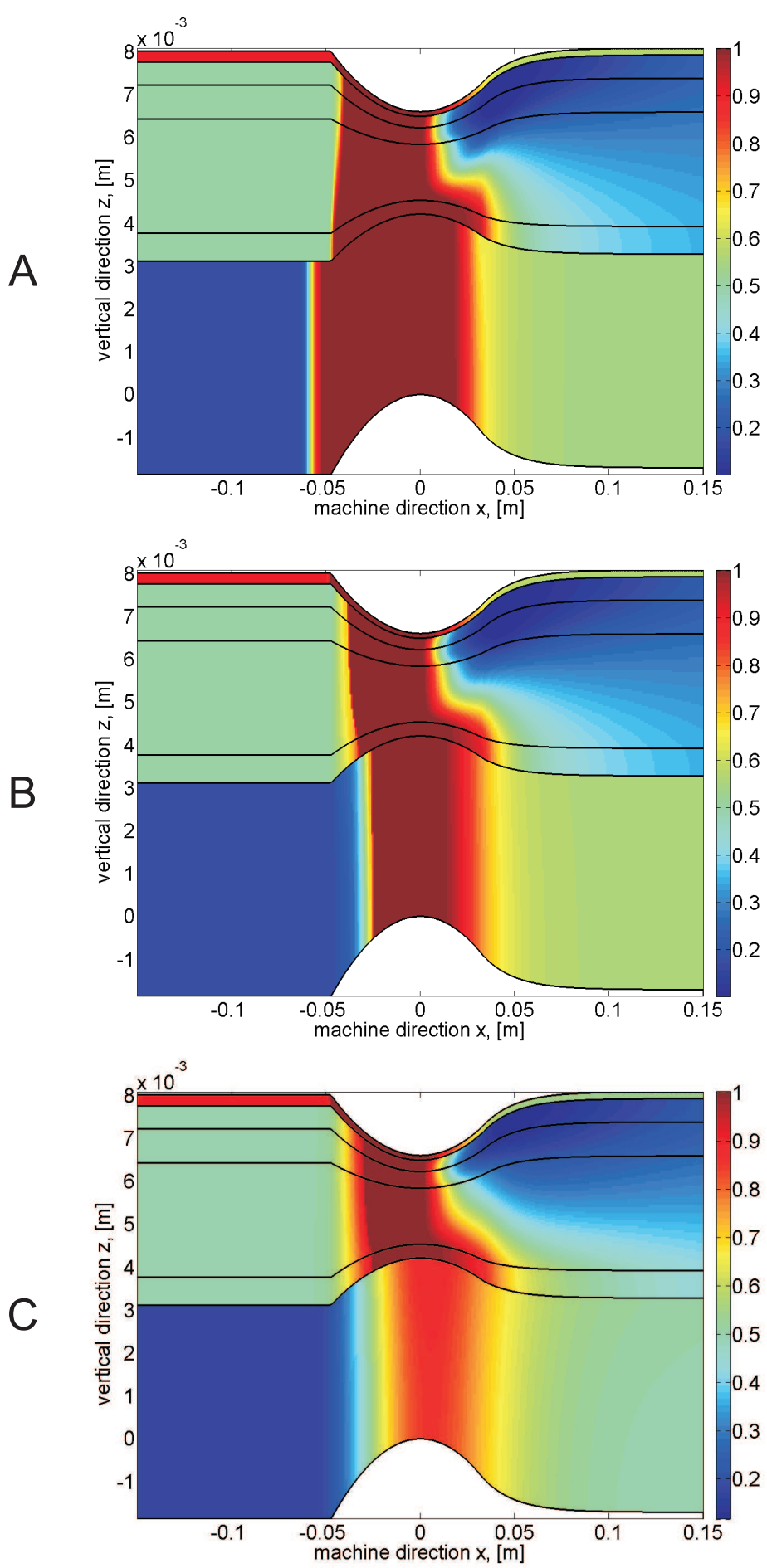

Fig. 20 Saturation for the test case 2 with $\tau$ equal to 0 (A), 10 (B) and $100 \mathrm{Pas}$ (C) 

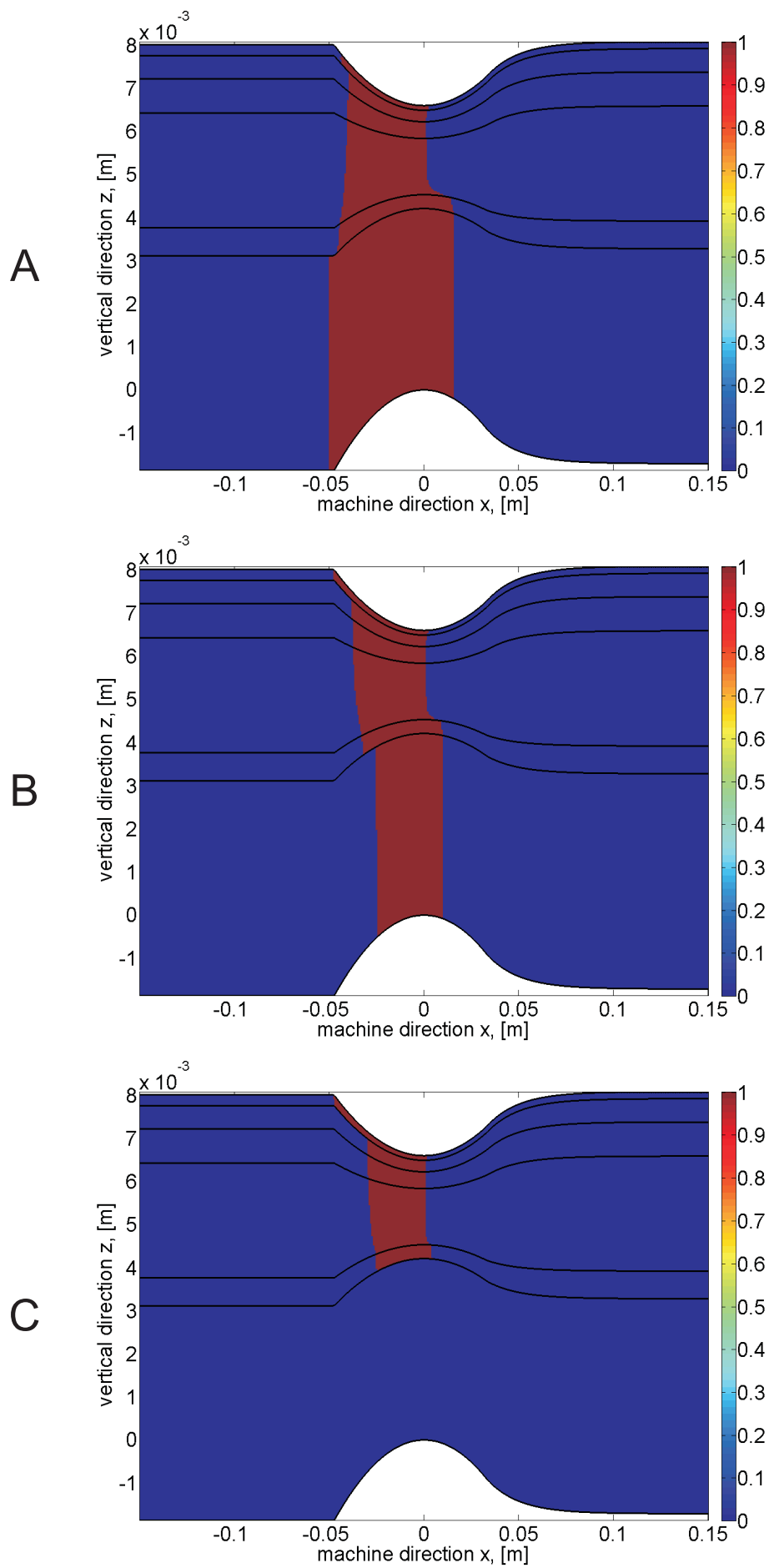

Fig. 21 Fully saturated zone for the test case 2 with $\tau$ equal to 0 (A), 10 (B) and $100 \mathrm{Pas}$ (C) 

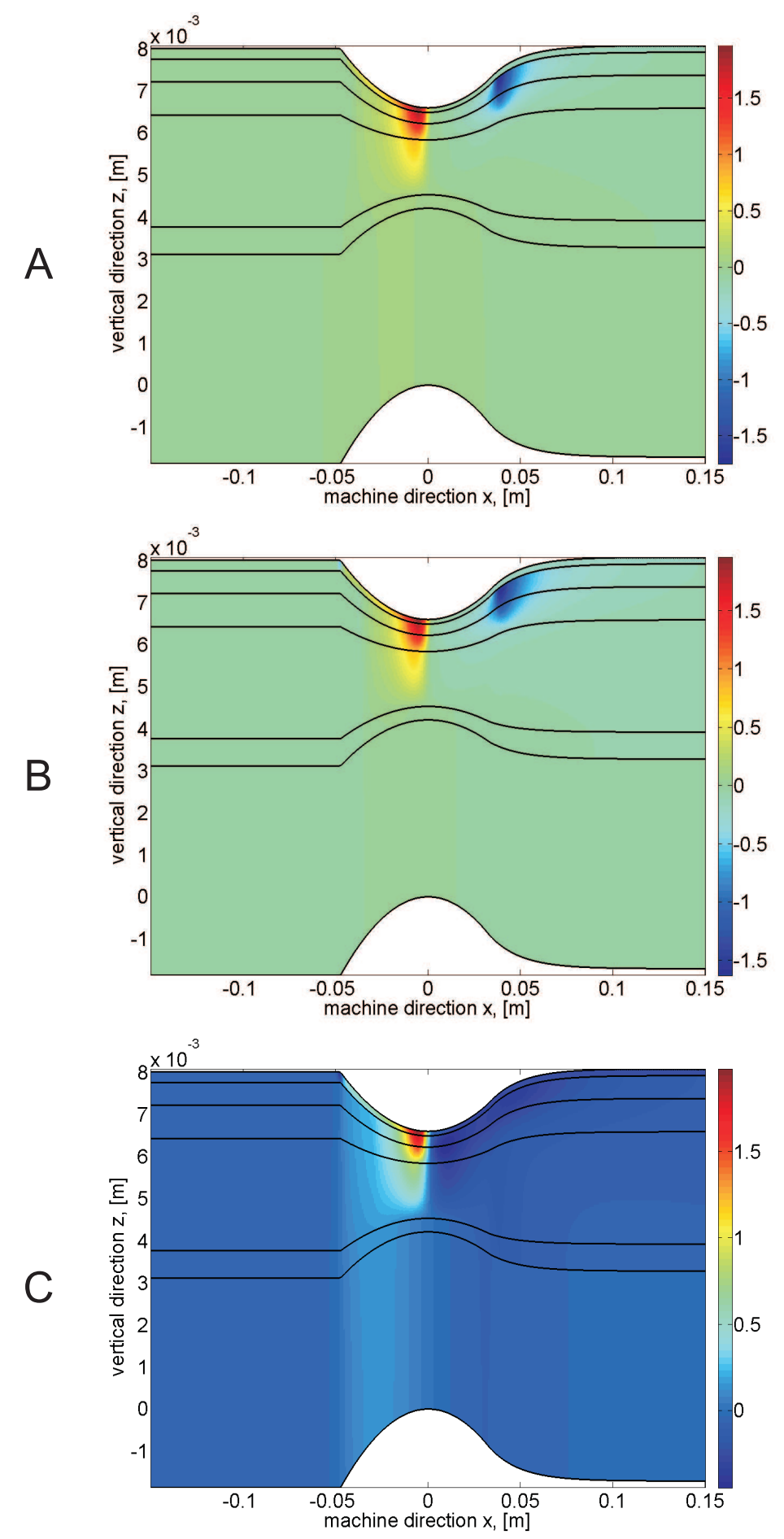

Fig. 22 Pressure for the test case 2 with $\tau$ equal to $0(\mathrm{~A}), 10(\mathrm{~B})$ and $100 \mathrm{~Pa} s(\mathrm{C})$ 


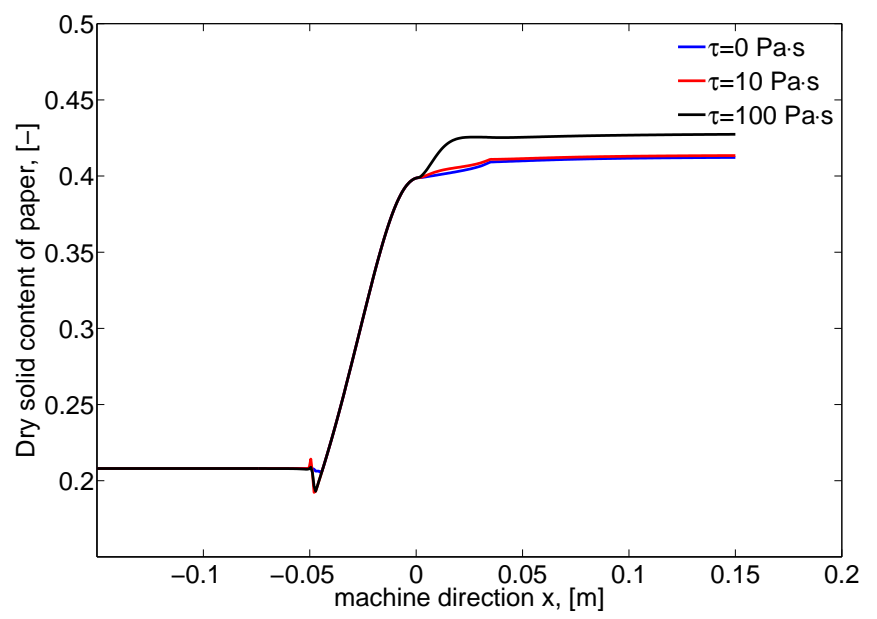

Fig. 23 Dry solid content of the paper for the test case 2 for different values of $\tau$ 

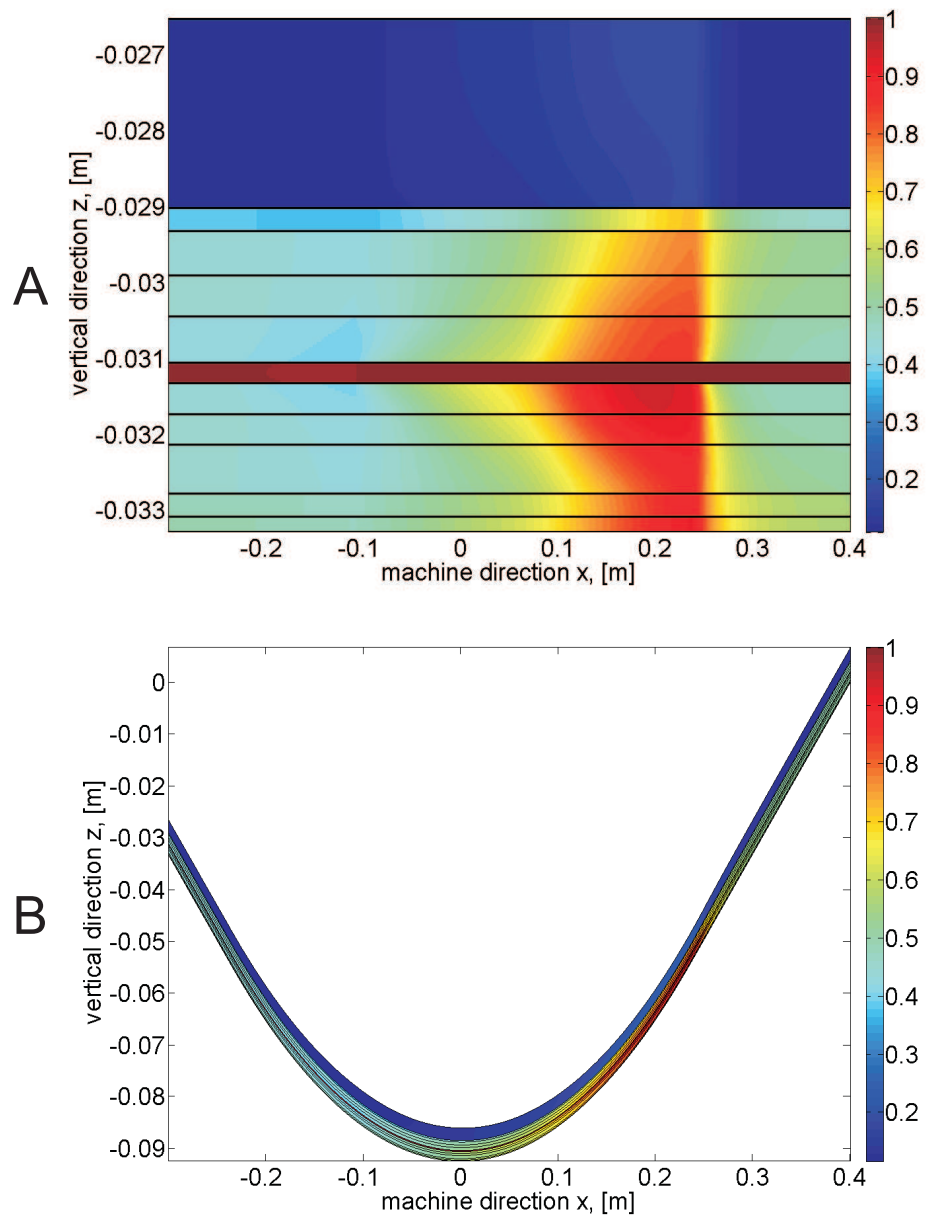

Fig. 24 Saturation for the test case 3 for different values of $\tau$ for the undeformed (A) and standard (B) computational domains 

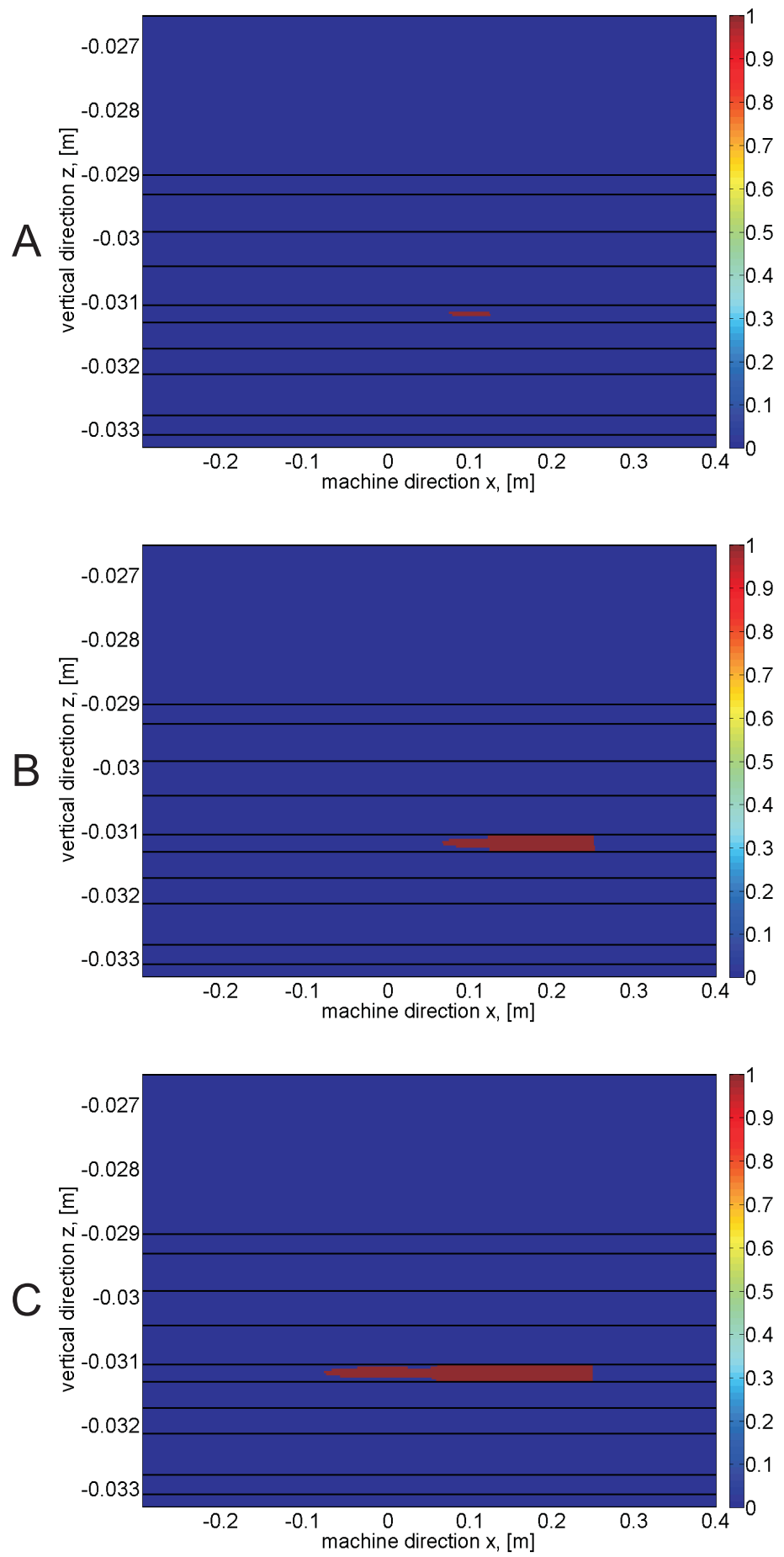

Fig. 25 Fully saturated zone for the test case 3 with $\tau$ equal to 0 (A), 10 (B) and $100 \mathrm{Pas}$ (C) 

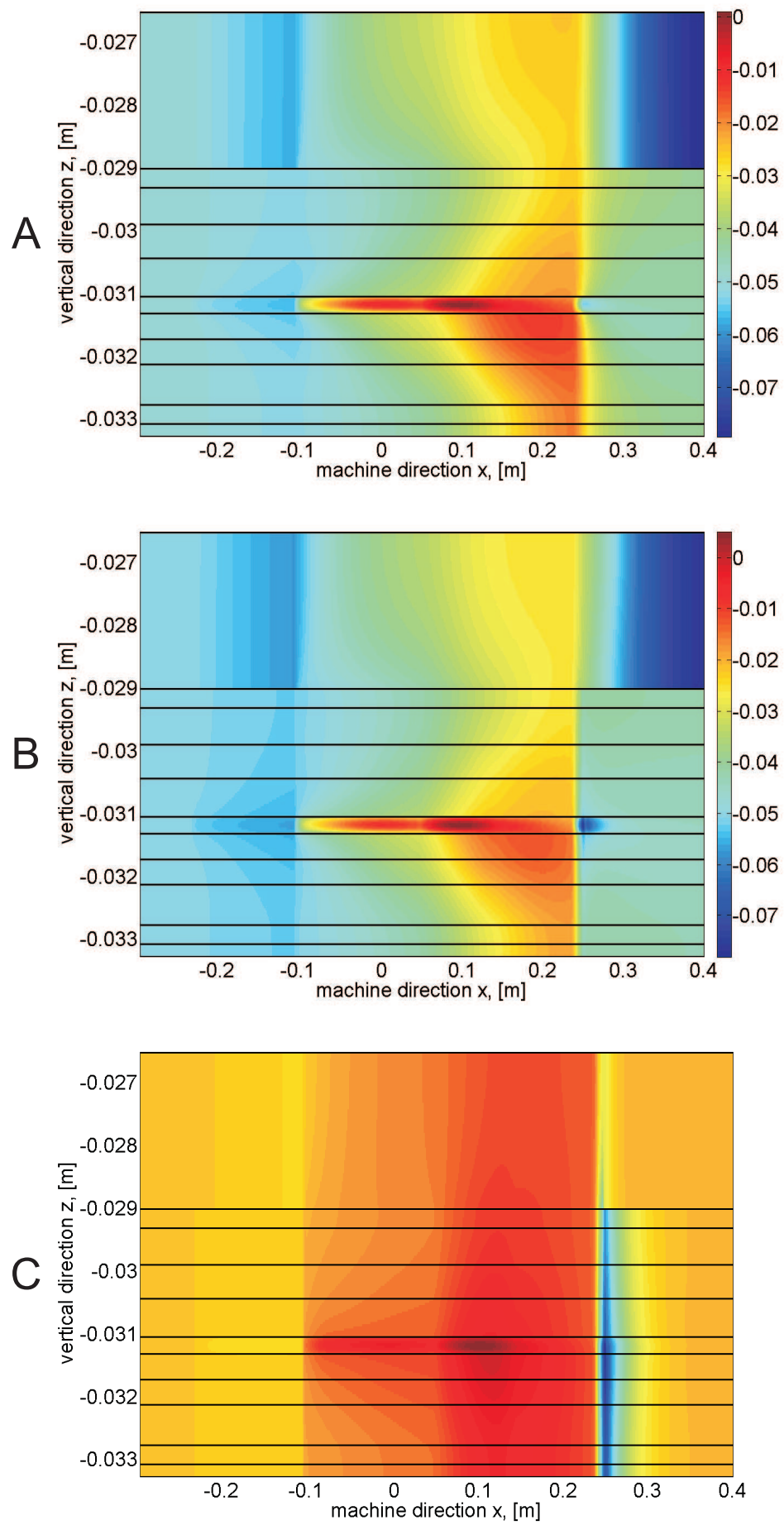

Fig. 26 Pressure for the test case 3 with $\tau$ equal to $0(\mathrm{~A}), 10(\mathrm{~B})$ and $100 \mathrm{~Pa} s(\mathrm{C})$ 


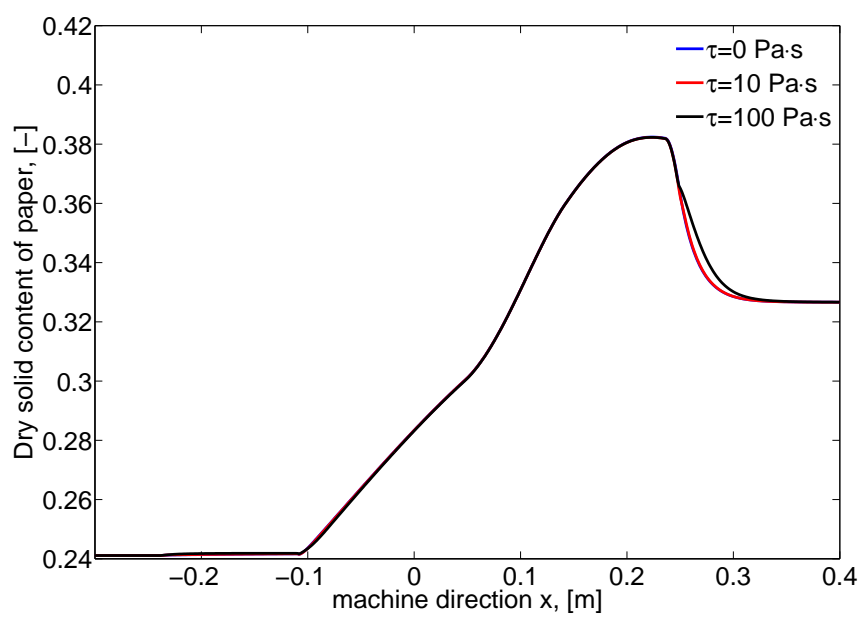

Fig. 27 Dry solid content for the test case 3 for different values of $\tau$ 

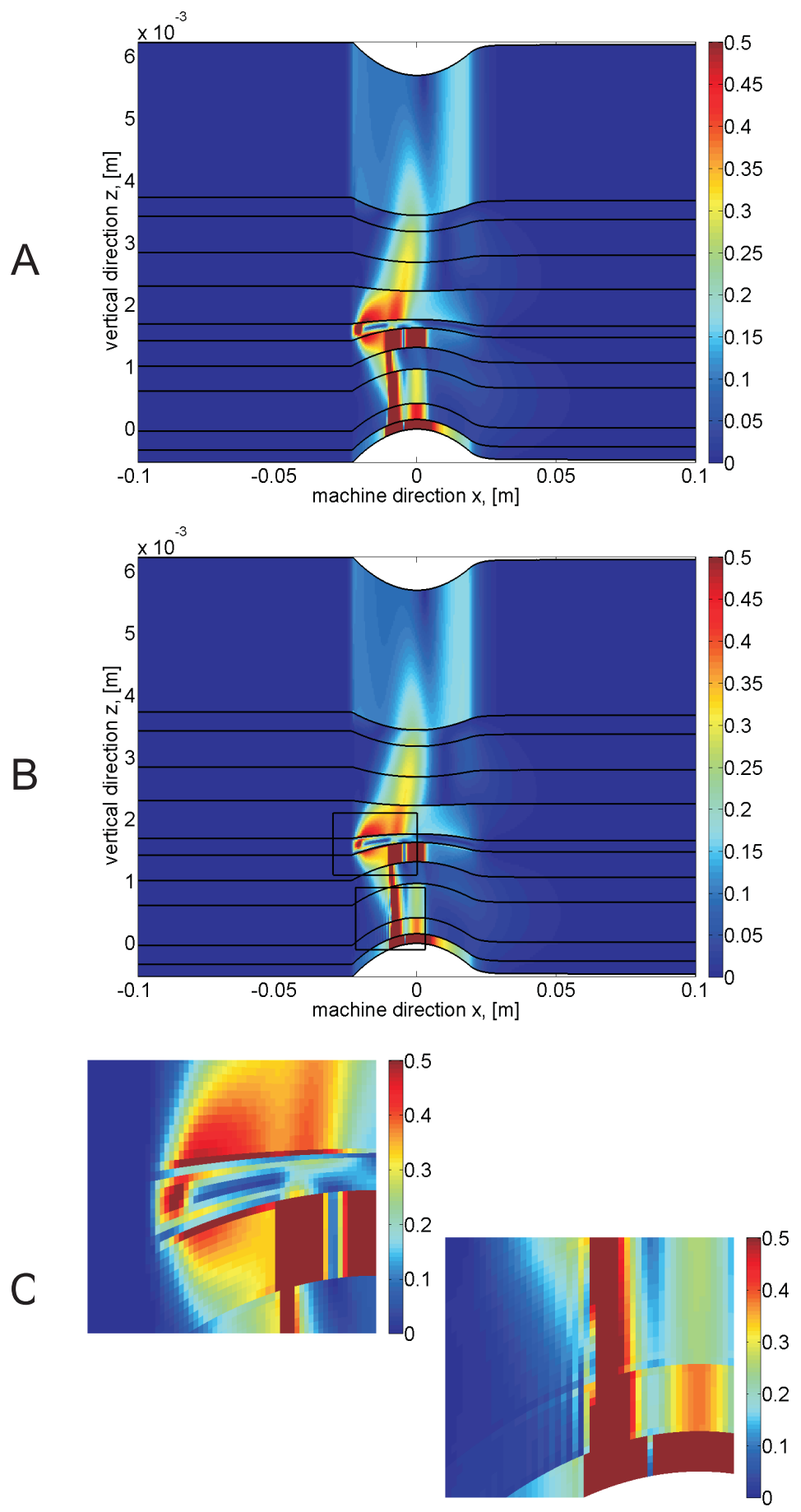

Fig. 28 Water velocity for the test case 1 with the static capillary pressure model obtained by the MPFA-O method (A) and by the FE method (B,C) 

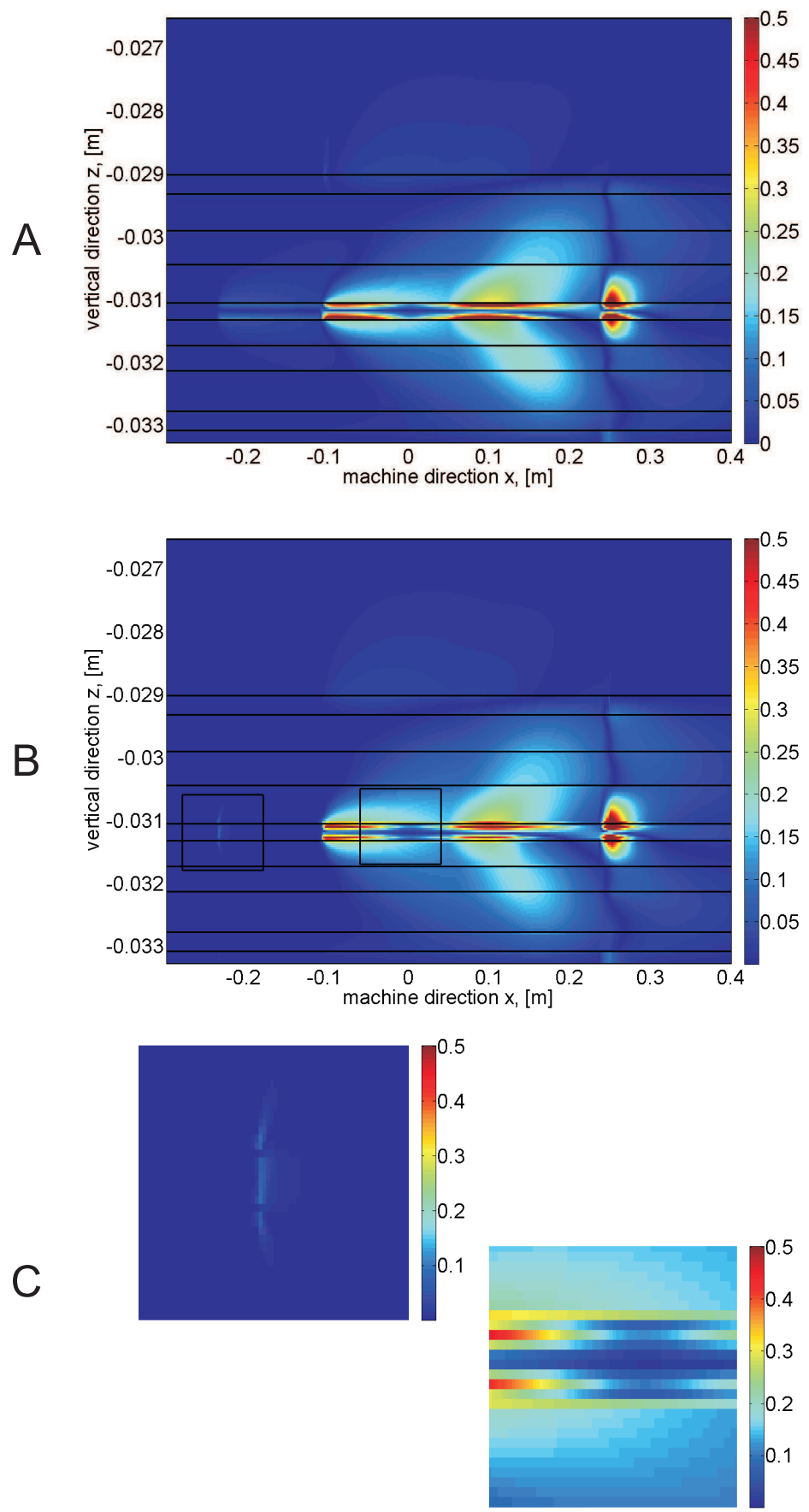

Fig. 29 Water velocity for the test case 3 with the static capillary pressure model obtained by the MPFA-O method (A) and by the FE method (B,C) 


\section{Published reports of the Fraunhofer ITWM}

The PDF-files of the following reports are available under:

\section{www.itwm.fraunhofer.de/de/ zentral_berichte/berichte}

1. D. Hietel, K. Steiner, J. Struckmeier A Finite - Volume Particle Method for Compressible Flows (19 pages, 1998)

2. M. Feldmann, S. Seibold

Damage Diagnosis of Rotors: Application of Hilbert Transform and Multi-Hypothesis Testing

Keywords: Hilbert transform, damage diagnosis,

Kalman filtering, non-linear dynamics

(23 pages, 1998)

3. Y. Ben-Haim, S. Seibold

Robust Reliability of Diagnostic MultiHypothesis Algorithms: Application to Rotating Machinery

Keywords: Robust reliability, convex models, Kalman filtering, multi-hypothesis diagnosis, rotating machinery, crack diagnosis

(24 pages, 1998)

\section{F.-Th. Lentes, N. Siedow}

Three-dimensional Radiative Heat Transfer in Glass Cooling Processes

(23 pages, 1998)

5. A. Klar, R. Wegener

A hierarchy of models for multilane vehicular traffic

Part I: Modeling

(23 pages, 1998)

Part II: Numerical and stochastic investigations (17 pages, 1998)

\section{A. Klar, N. Siedow}

Boundary Layers and Domain Decomposition for Radiative Heat Transfer and Diffusion Equations: Applications to Glass Manufacturing Processes

(24 pages, 1998)

7. I. Choquet

Heterogeneous catalysis modelling and numerical simulation in rarified gas flows Part l: Coverage locally at equilibrium (24 pages, 1998)

8. J. Ohser, B. Steinbach, C. Lang Efficient Texture Analysis of Binary Images (17 pages, 1998)

9. J. Orlik

Homogenization for viscoelasticity of the integral type with aging and shrinkage (20 pages, 1998)

10. J. Mohring

Helmholtz Resonators with Large Aperture (21 pages, 1998)
11. H. W. Hamacher, A. Schöbel On Center Cycles in Grid Graphs (15 pages, 1998)

12. H. W. Hamacher, K.-H. Küfer Inverse radiation therapy planning a multiple objective optimisation approach (14 pages, 1999)

13. C. Lang, J. Ohser, R. Hilfer On the Analysis of Spatial Binary Images (20 pages, 1999)

14. M. Junk

On the Construction of Discrete Equilibrium Distributions for Kinetic Schemes (24 pages, 1999)

15. M. Junk, S. V. Raghurame Rao

A new discrete velocity method for NavierStokes equations

(20 pages, 1999)

16. H. Neunzert

Mathematics as a Key to Key Technologies (39 pages, 1999)

17. J. Ohser, K. Sandau

Considerations about the Estimation of the Size Distribution in Wicksell's Corpuscle Problem

(18 pages, 1999)

18. E. Carrizosa, H. W. Hamacher, R. Klein, S. Nickel

Solving nonconvex planar location problems by finite dominating sets

Keywords: Continuous Location, Polyhedral Gauges, Finite Dominating Sets, Approximation, Sandwich Algorithm, Greedy Algorithm

(19 pages, 2000)

\section{A. Becker}

A Review on Image Distortion Measures Keywords: Distortion measure, human visual system (26 pages, 2000)

20. H. W. Hamacher, M. Labbé, S. Nickel, T. Sonneborn

Polyhedral Properties of the Uncapacitated Multiple Allocation Hub Location Problem Keywords: integer programming, hub location, facility location, valid inequalities, facets, branch and cut (21 pages, 2000)

21. H. W. Hamacher, A. Schöbel

Design of Zone Tariff Systems in Public Transportation

(30 pages, 2001)

22. D. Hietel, M. Junk, R. Keck, D. Teleaga The Finite-Volume-Particle Method for Conservation Laws (16 pages, 2001)

23. T. Bender, H. Hennes, J. Kalcsics, M. T. Melo, S. Nickel

Location Software and Interface with GIS and Supply Chain Management Keywords: facility location, software development, geographical information systems, supply chain management

(48 pages, 2001)
24. H. W. Hamacher, S. A. Tjandra Mathematical Modelling of Evacuation Problems: A State of Art (44 pages, 2001)

25. J. Kuhnert, S. Tiwari

Grid free method for solving the Poisson equation

Keywords: Poisson equation, Least squares method, Grid free method

(19 pages, 2001)

26. T. Götz, H. Rave, D. Reinel-Bitzer, K. Steiner, $\mathrm{H}$. Tiemeier

Simulation of the fiber spinning process Keywords: Melt spinning, fiber model, Lattice Boltzmann, CFD

(19 pages, 2001)

\section{A. Zemitis}

On interaction of a liquid film with an obstacle Keywords: impinging jets, liquid film, models, numerical solution, shape

22 pages, 2001)

\section{I. Ginzburg, K. Steiner}

Free surface lattice-Boltzmann method to model the filling of expanding cavities by Bingham Fluids

Keywords: Generalized LBE, free-surface phenomena interface boundary conditions, filling processes, Bing ham viscoplastic model, regularized models

(22 pages, 2001)

\section{H. Neunzert}

"Denn nichts ist für den Menschen als Menschen etwas wert, was er nicht mit Leidenschaft tun kann

Vortrag anlässlich der Verleihung des Akademiepreises des Landes RheinlandPfalz am 21.11.2001

Keywords: Lehre, Forschung, angewandte Mathematik, Mehrskalenanalyse, Strömungsmechanik (18 pages, 2001)

30. J. Kuhnert, S. Tiwari

Finite pointset method based on the projection method for simulations of the incompressible Navier-Stokes equations

Keywords: Incompressible Navier-Stokes equations, Meshfree method, Projection method, Particle scheme, Least squares approximation

AMS subject classification: 76D05, 76M28

(25 pages, 2001)

31. R. Korn, M. Krekel

Optimal Portfolios with Fixed Consumption or Income Streams

Keywords: Portfolio optimisation, stochastic control, HJB equation, discretisation of control problems (23 pages, 2002)

32. M. Krekel

Optimal portfolios with a loan dependent credit spread

Keywords: Portfolio optimisation, stochastic control, HJB equation, credit spread, log utility, power utility, non-linear wealth dynamics

(25 pages, 2002)

33. J. Ohser, W. Nagel, K. Schladitz

The Euler number of discretized sets - on the choice of adjacency in homogeneous lattices Keywords: image analysis, Euler number, neighborhod relationships, cuboidal lattice

(32 pages, 2002) 
34. I. Ginzburg, K. Steiner

Lattice Boltzmann Model for Free-Surface flow and Its Application to Filling Process in Casting

Keywords: Lattice Boltzmann models; free-surface phenomena; interface boundary conditions; filling processes; injection molding; volume of fluid method; interface boundary conditions; advection-schemes; upwind-schemes

(54 pages, 2002)

35. M. Günther, A. Klar, T. Materne, R. Wegener

Multivalued fundamental diagrams and stop and go waves for continuum traffic equations Keywords: traffic flow, macroscopic equations, kinetic derivation, multivalued fundamental diagram, stop and go waves, phase transitions

(25 pages, 2002)

36. S. Feldmann, P. Lang, D. Prätzel-Wolters Parameter influence on the zeros of network determinants

Keywords: Networks, Equicofactor matrix polynomials, Realization theory, Matrix perturbation theory

(30 pages, 2002)

\section{K. Koch, J. Ohser, K. Schladitz} Spectral theory for random closed sets and estimating the covariance via frequency space

Keywords: Random set, Bartlett spectrum, fast Fourier transform, power spectrum

(28 pages, 2002)

\section{D. d'Humières, I. Ginzburg}

Multi-reflection boundary conditions for lattice Boltzmann models

Keywords: lattice Boltzmann equation, boudary condistions, bounce-back rule, Navier-Stokes equation

(72 pages, 2002)

\section{R. Korn}

\section{Elementare Finanzmathematik}

Keywords: Finanzmathematik, Aktien, Optionen, Portfolio-Optimierung, Börse, Lehrerweiterbildung, Mathematikunterricht

(98 pages, 2002)

40. J. Kallrath, M. C. Müller, S. Nickel

Batch Presorting Problems:

Models and Complexity Results

Keywords: Complexity theory, Integer programming,

Assigment, Logistics

(19 pages, 2002)

\section{J. Linn}

On the frame-invariant description of the phase space of the Folgar-Tucker equation Key words: fiber orientation, Folgar-Tucker equation, injection molding

(5 pages, 2003)

\section{T. Hanne, S. Nickel}

A Multi-Objective Evolutionary Algorithm for Scheduling and Inspection Planning in Software Development Projects

Key words: multiple objective programming, project management and scheduling, software development, evolutionary algorithms, efficient set

(29 pages, 2003)

43. T. Bortfeld, K.-H. Küfer, M. Monz,

A. Scherrer, C. Thieke, H. Trinkaus

Intensity-Modulated Radiotherapy - A Large Scale Multi-Criteria Programming Problem
Keywords: multiple criteria optimization, representative systems of Pareto solutions, adaptive triangulation, clustering and disaggregation techniques, visualization of Pareto solutions, medical physics, external beam radiotherapy planning, intensity modulated radiotherapy (31 pages, 2003)

\section{T. Halfmann, T. Wichmann}

Overview of Symbolic Methods in Industrial Analog Circuit Design

Keywords: $C A D$, automated analog circuit design, sym bolic analysis, computer algebra, behavioral modeling, system simulation, circuit sizing, macro modeling, differential-algebraic equations, index

(17 pages, 2003)

\section{S. E. Mikhailov, J. Orlik}

Asymptotic Homogenisation in Strength and Fatigue Durability Analysis of Composites

Keywords: multiscale structures, asymptotic homogenization, strength, fatigue, singularity, non-local conditions

(14 pages, 2003)

46. P. Domínguez-Marín, P. Hansen, N. Mladenovic, S. Nickel Heuristic Procedures for Solving the Discrete Ordered Median Problem Keywords: genetic algorithms, variable neighborhood search, discrete facility location

(31 pages, 2003)

47. N. Boland, P. Domínguez-Marín, S. Nickel, J. Puerto

Exact Procedures for Solving the Discrete Ordered Median Problem

Keywords: discrete location, Integer programming

(41 pages, 2003)

\section{S. Feldmann, P. Lang}

Padé-like reduction of stable discrete linear systems preserving their stability Keywords: Discrete linear systems, model reduction, stability, Hankel matrix, Stein equation

(16 pages, 2003)

49. J. Kallrath, S. Nicke

A Polynomial Case of the Batch Presorting Problem

Keywords: batch presorting problem, online optimization competetive analysis, polynomial algorithms, logistics (17 pages, 2003)

\section{T. Hanne, H. L. Trinkaus}

knowCube for MCDM -

Visual and Interactive Support for Multicriteria Decision Making

Key words: Multicriteria decision making, knowledge management, decision support systems, visual interfaces, interactive navigation, real-life applications. (26 pages, 2003)

51. O. lliev, V. Laptev

On Numerical Simulation of Flow Through Oil Filters

Keywords: oil filters, coupled flow in plain and porous media, Navier-Stokes, Brinkman, numerical simulation (8 pages, 2003)

52. W. Dörfler, O. Iliev, D. Stoyanov, D. Vassileva On a Multigrid Adaptive Refinement Solver for Saturated Non-Newtonian Flow in Porous Media

Keywords: Nonlinear multigrid, adaptive refinement, non-Newtonian flow in porous media

(17 pages, 2003)
53. S. Kruse

On the Pricing of Forward Starting Options under Stochastic Volatility

Keywords: Option pricing, forward starting options, Heston model, stochastic volatility, cliquet options (11 pages, 2003)

54. O. Iliev, D. Stoyanov

Multigrid - adaptive local refinement solver for incompressible flows

Keywords: Navier-Stokes equations, incompressible flow projection-type splitting, SIMPLE, multigrid methods, adaptive local refinement, lid-driven flow in a cavity (37 pages, 2003)

\section{V. Starikovicius}

The multiphase flow and heat transfer in porous media

Keywords: Two-phase flow in porous media, various formulations, global pressure, multiphase mixture model, numerical simulation

(30 pages, 2003)

\section{P. Lang, A. Sarishvili, A. Wirsen}

Blocked neural networks for knowledge extraction in the software development process Keywords: Blocked Neural Networks, Nonlinear Regression, Knowledge Extraction, Code Inspection (21 pages, 2003)

\section{H. Knaf, P. Lang, S. Zeiser}

Diagnosis aiding in Regulation

Thermography using Fuzzy Logic

Keywords: fuzzy logic, knowledge representation, expert system

(22 pages, 2003)

58. M. T. Melo, S. Nickel, F. Saldanha da Gama Largescale models for dynamic multicommodity capacitated facility location Keywords: supply chain management, strategic planning, dynamic location, modeling (40 pages, 2003)

\section{J. Orlik}

Homogenization for contact problems with periodically rough surfaces

Keywords: asymptotic homogenization, contact problems (28 pages, 2004)

60. A. Scherrer, K.-H. Küfer, M. Monz,

F. Alonso, T. Bortfeld

IMRT planning on adaptive volume structures - a significant advance of computational complexity

Keywords: Intensity-modulated radiation therapy (IMRT), inverse treatment planning, adaptive volume structures, hierarchical clustering, local refinement, adaptive clustering, convex programming, mesh generation, multi-grid methods

(24 pages, 2004)

61. D. Kehrwald

Parallel lattice Boltzmann simulation of complex flows

Keywords: Lattice Boltzmann methods, parallel computing, microstructure simulation, virtual material design, pseudo-plastic fluids, liquid composite moulding (12 pages, 2004)

62. O. Iliev, J. Linn, M. Moog, D. Niedziela, V. Starikovicius

On the Performance of Certain Iterative Solvers for Coupled Systems Arising in Discretization of Non-Newtonian Flow Equations 
Keywords: Performance of iterative solvers, Preconditioners, Non-Newtonian flow

(17 pages, 2004)

\section{R. Ciegis, O. Iliev, S. Rief, K. Steiner}

On Modelling and Simulation of Different Regimes for Liquid Polymer Moulding Keywords: Liquid Polymer Moulding, Modelling, Simulation, Infiltration, Front Propagation, non-Newtonian flow in porous media

(43 pages, 2004)

\section{T. Hanne, H. Neu}

Simulating Human Resources in

\section{Software Development Processes}

Keywords: Human resource modeling, software process, productivity, human factors, learning curve

(14 pages, 2004)

\section{O. Iliev, A. Mikelic, P. Popov}

Fluid structure interaction problems in de-

formable porous media: Toward permeability of deformable porous media

Keywords: fluid-structure interaction, deformable porous media, upscaling, linear elasticity, stokes, finite elements

(28 pages, 2004)

66. F. Gaspar, O. Iliev, F. Lisbona, A. Naumovich, P. Vabishchevich

On numerical solution of 1-D poroelasticity equations in a multilayered domain Keywords: poroelasticity, multilayered material, finite volume discretization, MAC type grid

(41 pages, 2004)

67. J. Ohser, K. Schladitz, K. Koch, M. Nöthe Diffraction by image processing and its application in materials science

Keywords: porous microstructure, image analysis, random set, fast Fourier transform, power spectrum, Bartlett spectrum

(13 pages, 2004)

\section{H. Neunzert}

\section{Mathematics as a Technology: Challenges} for the next 10 Years

Keywords: applied mathematics, technology, modelling, simulation, visualization, optimization, glass processing, spinning processes, fiber-fluid interaction, trubulence effects, topological optimization, multicriteria optimization, Uncertainty and Risk, financial mathematics, Malliavin calculus, Monte-Carlo methods, virtual material design, filtration, bio-informatics, system biology

(29 pages, 2004)

69. R. Ewing, O. lliev, R. Lazarov, A. Naumovich On convergence of certain finite difference discretizations for $1 D$ poroelasticity interface problems

Keywords: poroelasticity, multilayered material, finite volume discretizations, MAC type grid, error estimates (26 pages, 2004 )

70. W. Dörfler, O. Iliev, D. Stoyanov, D. Vassileva On Efficient Simulation of Non-Newtonian Flow in Saturated Porous Media with a Multigrid Adaptive Refinement Solver Keywords: Nonlinear multigrid, adaptive renement non-Newtonian in porous media (25 pages, 2004)

\section{J. Kalcsics, S. Nickel, M. Schröde}

Towards a Unified Territory Design Approach - Applications, Algorithms and GIS Integration Keywords: territory desgin, political districting, sales territory alignment, optimization algorithms, Geographical Information Systems

(40 pages, 2005)
72. K. Schladitz, S Peters, D Reinel-Bitzer, A. Wiegmann, J. Ohser

Design of acoustic trim based on geometric modeling and flow simulation for non-woven Keywords: random system of fibers, Poisson line process, flow resistivity, acoustic absorption, Lattice-Boltzmann method, non-woven (21 pages, 2005)

\section{V. Rutka, A. Wiegmann}

Explicit Jump Immersed Interface Method for virtual material design of the effective elastic moduli of composite materials Keywords: virtual material design, explicit jump immersed interface method, effective elastic moduli, composite materials

(22 pages, 2005)

\section{T. Hanne}

Eine Übersicht zum Scheduling von Baustellen Keywords: Projektplanung, Scheduling, Bauplanung, Bauindustrie

(32 pages, 2005)

75. J. Linn

The Folgar-Tucker Model as a Differetial Algebraic System for Fiber Orientation Calculation

Keywords: fiber orientation, Folgar-Tucker model, invariants, algebraic constraints, phase space, trace stability

(15 pages, 2005)

76. M. Speckert, K. Dreßler, H. Mauch, A. Lion, G. J. Wierda

Simulation eines neuartigen Prüfsystems für Achserprobungen durch MKS-Modellierung einschließlich Regelung Keywords: virtual test rig, suspension testing, multibody simulation, modeling hexapod test rig, opti mization of test rig configuration

(20 pages, 2005)

77. K.-H. Küfer, M. Monz, A. Scherrer, P. Süss, F. Alonso, A. S. A. Sultan, Th. Bortfeld,

D. Craft, Chr. Thieke

Multicriteria optimization in intensity modulated radiotherapy planning Keywords: multicriteria optimization, extreme solutions, real-time decision making, adaptive approxima tion schemes, clustering methods, IMRT planning, reverse engineering

(51 pages, 2005)

78. S. Amstutz, H. Andrä

A new algorithm for topology optimization using a level-set method

Keywords: shape optimization, topology optimization, topological sensitivity, level-set

(22 pages, 2005)

\section{N. Ettrich}

Generation of surface elevation models for urban drainage simulation

Keywords: Flooding, simulation, urban elevation models, laser scanning

(22 pages, 2005

80. H. Andrä, J. Linn, I. Matei, I. Shklyar, K. Steiner, E. Teichmann

OPTCAST - Entwicklung adäquater Struk turoptimierungsverfahren für Gießereien Technischer Bericht (KURZFASSUNG) Keywords: Topologieoptimierung, Level-Set-Methode Gießprozesssimulation, Gießtechnische Restriktionen, CAE-Kette zur Strukturoptimierung

(77 pages, 2005)
81. N. Marheineke, R. Wegener Fiber Dynamics in Turbulent Flows Part I: General Modeling Framework Keywords: fiber-fluid interaction; Cosserat rod; turbuence modeling: Kolmogorov's energy spectrum; double-velocity correlations; differentiable Gaussian fields (20 pages, 2005)

\section{Part II: Specific Taylor Drag}

Keywords: flexible fibers; $k-\varepsilon$ turbulence model; fiber-turbulence interaction scales; air drag; random Gaussian aerodynamic force; white noise; stochastic differential equations; ARMA process

(18 pages, 2005)

\section{C. H. Lampert, O. Wirjadi}

An Optimal Non-Orthogonal Separation of the Anisotropic Gaussian Convolution Filter Keywords: Anisotropic Gaussian filter, linear filtering, or entation space, $n D$ image processing, separable filters (25 pages, 2005)

83. H. Andrä, D. Stoyanov

Error indicators in the parallel finite element solver for linear elasticity DDFEM Keywords: linear elasticity, finite element method, hierarchical shape functions, domain decom-position, parallel implementation, a posteriori error estimates (21 pages, 2006)

84. M. Schröder, I. Solchenbach Optimization of Transfer Quality in Regional Public Transit

Keywords: public transit, transfer quality, quadratic assignment problem

(16 pages, 2006

85. A. Naumovich, F. J. Gaspar

On a multigrid solver for the three-dimensional Biot poroelasticity system in multilayered domains

Keywords: poroelasticity, interface problem, multigrid, operator-dependent prolongation

(11 pages, 2006)

86. S. Panda, R. Wegener, N. Marheineke Slender Body Theory for the Dynamics of Curved Viscous Fibers

Keywords: curved viscous fibers; fluid dynamics; NavierStokes equations; free boundary value problem; asymp totic expansions; slender body theory

(14 pages, 2006)

87. E. Ivanov, H. Andrä, A. Kudryavtsev Domain Decomposition Approach for Automatic Parallel Generation of Tetrahedral Grids Key words: Grid Generation, Unstructured Grid, Delau nay Triangulation, Parallel Programming, Domain Decomposition, Load Balancing

(18 pages, 2006)

88. S. Tiwari, S. Antonov, D. Hietel, J. Kuhnert, R. Wegener

A Meshfree Method for Simulations of In teractions between Fluids and Flexible Structures

Key words: Meshfree Method, FPM, Fluid Structure Interaction, Sheet of Paper, Dynamical Coupling (16 pages, 2006)

89. R. Ciegis, O. Iliev, V. Starikovicius, K. Steine Numerical Algorithms for Solving Problems of Multiphase Flows in Porous Media Keywords: nonlinear algorithms, finite-volume method software tools, porous media, flows

16 pages, 2006) 
90. D. Niedziela, O. Iliev, A. Latz

On 3D Numerical Simulations of Viscoelastic Fluids

Keywords: non-Newtonian fluids, anisotropic viscosity, integral constitutive equation

(18 pages, 2006)

\section{A. Winterfeld}

Application of general semi-infinite Programming to Lapidary Cutting Problems Keywords: large scale optimization, nonlinear programming, general semi-infinite optimization, design centering, clustering

(26 pages, 2006)

\section{J. Orlik, A. Ostrovska}

Space-Time Finite Element Approximation and Numerical Solution of Hereditary Linear Viscoelasticity Problems

Keywords: hereditary viscoelasticity; kern approximation by interpolation; space-time finite element approximation, stability and a priori estimate

(24 pages, 2006)

93. V. Rutka, A. Wiegmann, H. Andrä EJIIM for Calculation of effective Elastic Moduli in 3D Linear Elasticity

Keywords: Elliptic $P D E$, linear elasticity, irregular domain, finite differences, fast solvers, effective elastic moduli

(24 pages, 2006)

\section{A. Wiegmann, A. Zemitis}

EJ-HEAT: A Fast Explicit Jump Harmonic Averaging Solver for the Effective Heat Conductivity of Composite Materials Keywords: Stationary heat equation, effective thermal conductivity, explicit jump, discontinuous coefficients, virtual material design, microstructure simulation, EJ-HEAT

(21 pages, 2006)

\section{A. Naumovich}

On a finite volume discretization of the three-dimensional Biot poroelasticity system in multilayered domains

Keywords: Biot poroelasticity system, interface problems, finite volume discretization, finite difference method (21 pages, 2006)

\section{M. Krekel, J. Wenzel}

A unified approach to Credit Default Swaption and Constant Maturity Credit Default Swap valuation

Keywords: LIBOR market model, credit risk, Credit Default Swaption, Constant Maturity Credit Default Swapmethod

(43 pages, 2006)

\section{A. Dreyer}

\section{Interval Methods for Analog Circiuts}

Keywords: interval arithmetic, analog circuits, tolerance analysis, parametric linear systems, frequency response, symbolic analysis, CAD, computer algebra

(36 pages, 2006)

98. N. Weigel, S. Weihe, G. Bitsch, K. Dreßler Usage of Simulation for Design and Optimization of Testing

Keywords: Vehicle test rigs, MBS, control, hydraulics,

testing philosophy

(14 pages, 2006)

99. H. Lang, G. Bitsch, K. Dreßler, M. Speckert Comparison of the solutions of the elastic and elastoplastic boundary value problems
Keywords: Elastic BVP, elastoplastic BVP, variational inequalities, rate-independency, hysteresis, linear kinematic hardening, stop- and play-operator

(21 pages, 2006)

100. M. Speckert, K. Dreßler, H. Mauch MBS Simulation of a hexapod based suspension test rig

Keywords: Test rig, MBS simulation, suspension,

hydraulics, controlling, design optimization

(12 pages, 2006)

101. S. Azizi Sultan, K.-H. Küfer

A dynamic algorithm for beam orientations in multicriteria IMRT planning

Keywords: radiotherapy planning, beam orientation optimization, dynamic approach, evolutionary algorithm, global optimization

(14 pages, 2006)

102. T. Götz, A. Klar, N. Marheineke, R. Wegener A Stochastic Model for the Fiber Lay-down Process in the Nonwoven Production Keywords: fiber dynamics, stochastic Hamiltonian system, stochastic averaging

(17 pages, 2006)

103. Ph. Süss, K.-H. Küfer

Balancing control and simplicity: a variable aggregation method in intensity modulated radiation therapy planning

Keywords: IMRT planning, variable aggregation, clustering methods

(22 pages, 2006)

104. A. Beaudry, G. Laporte, T. Melo, S. Nickel Dynamic transportation of patients in hospitals

Keywords: in-house hospital transportation, dial-a-ride, dynamic mode, tabu search

(37 pages, 2006)

105. Th. Hanne

Applying multiobjective evolutionary algorithms in industrial projects

Keywords: multiobjective evolutionary algorithms, discrete optimization, continuous optimization, electronic circuit design, semi-infinite programming, scheduling (18 pages, 2006)

106. J. Franke, S. Halim

Wild bootstrap tests for comparing signals and images

Keywords: wild bootstrap test, texture classification, textile quality control, defect detection, kernel estimate, nonparametric regression

(13 pages, 2007)

107. Z. Drezner, S. Nickel

Solving the ordered one-median problem in the plane

Keywords: planar location, global optimization, ordered median, big triangle small triangle method, bounds, numerical experiments

(21 pages, 2007)

108. Th. Götz, A. Klar, A. Unterreiter, R. Wegener

Numerical evidance for the non-existing of solutions of the equations desribing rotational fiber spinning

Keywords: rotational fiber spinning, viscous fibers, boundary value problem, existence of solutions (11 pages, 2007)
109. Ph. Süss, K.-H. Küfer

Smooth intensity maps and the BortfeldBoyer sequencer

Keywords: probabilistic analysis, intensity modulated radiotherapy treatment (IMRT), IMRT plan application step-and-shoot sequencing

(8 pages, 2007)

110. E. Ivanov, O. Gluchshenko, H. Andrä, A. Kudryavtsev

Parallel software tool for decomposing and meshing of $3 d$ structures

Keywords: a-priori domain decomposition, unstructured grid, Delaunay mesh generation

(14 pages, 2007)

111. O. Iliev, R. Lazarov, J. Willems Numerical study of two-grid preconditioners for 1d elliptic problems with highly oscillating discontinuous coefficients Keywords: two-grid algorithm, oscillating coefficients, preconditioner

(20 pages, 2007)

112. L. Bonilla, T. Götz, A. Klar, N. Marheineke, R. Wegener

Hydrodynamic limit of the Fokker-Planckequation describing fiber lay-down processes

Keywords: stochastic dierential equations, FokkerPlanck equation, asymptotic expansion, Ornstein-

Uhlenbeck process

(17 pages, 2007)

\section{S. Rief}

Modeling and simulation of the pressing section of a paper machine

Keywords: paper machine, computational fluid dynamics, porous media

(41 pages, 2007)

114. R. Ciegis, O. Iliev, Z. Lakdawala

On parallel numerical algorithms for simulating industrial filtration problems

Keywords: Navier-Stokes-Brinkmann equations, finite volume discretization method, SIMPLE, parallel computing, data decomposition method

(24 pages, 2007)

\section{N. Marheineke, R. Wegener}

Dynamics of curved viscous fibers with surface tension

Keywords: Slender body theory, curved viscous bers with surface tension, free boundary value problem (25 pages, 2007)

116. S. Feth, J. Franke, M. Speckert Resampling-Methoden zur mse-Korrektur und Anwendungen in der Betriebsfestigkeit Keywords: Weibull, Bootstrap, Maximum-Likelihood, Betriebsfestigkeit

(16 pages, 2007)

117. H. Knaf

Kernel Fisher discriminant functions - a concise and rigorous introduction

Keywords: wild bootstrap test, texture classification, textile quality control, defect detection, kernel estimate, nonparametric regression

(30 pages, 2007)

118. O. Iliev, I. Rybak

On numerical upscaling for flows in heterogeneous porous media 
Keywords: numerical upscaling heterogeneous porous media, single phase flow, Darcy's law, multiscale problem, effective permeability, multipoint flux approximation, anisotropy

(17 pages, 2007)

\section{O. Iliev, I. Rybak}

On approximation property of multipoint flux approximation method

Keywords: Multipoint flux approximation, finite volume method, elliptic equation, discontinuous tensor coeffi-

cients, anisotropy

(15 pages, 2007)

120. O. Iliev, I. Rybak, J. Willems

On upscaling heat conductivity for a class of industrial problems

Keywords: Multiscale problems, effective heat conductivity, numerical upscaling, domain decomposition

(21 pages, 2007)

121. R. Ewing, O. Iliev, R. Lazarov, I. Rybak On two-level preconditioners for flow in porous media

Keywords: Multiscale problem, Darcy's law, single phase flow, anisotropic heterogeneous porous media, numerical upscaling, multigrid, domain decomposition, efficient preconditioner

(18 pages, 2007)

122. M. Brickenstein, A. Dreyer

POLYBORI: A Gröbner basis framework for Boolean polynomials

Keywords: Gröbner basis, formal verification, Boolean polynomials, algebraic cryptoanalysis, satisfiability

(23 pages, 2007)

\section{O. Wirjad}

Survey of $3 d$ image segmentation methods Keywords: image processing, 3d, image segmentation, binarization

(20 pages, 2007)

\section{S. Zeytun, A. Gupta}

A Comparative Study of the Vasicek and the CIR Model of the Short Rate

Keywords: interest rates, Vasicek model, CIR-model,

calibration, parameter estimation

(17 pages, 2007)

\section{G. Hanselmann, A. Sarishvili}

Heterogeneous redundancy in software quality prediction using a hybrid Bayesian approach

Keywords: reliability prediction, fault prediction, nonhomogeneous poisson process, Bayesian model averaging

(17 pages, 2007)

126. V. Maag, M. Berger, A. Winterfeld, K.-H Küfer

A novel non-linear approach to minimal area rectangular packing

Keywords: rectangular packing, non-overlapping constraints, non-linear optimization, regularization, relaxation

(18 pages, 2007)

127. M. Monz, K.-H. Küfer, T. Bortfeld, C. Thieke Pareto navigation - systematic multi-criteria-based IMRT treatment plan determination

Keywords: convex, interactive multi-objective optimization, intensity modulated radiotherapy planning

(15 pages, 2007)
128. M. Krause A Scherrer

On the role of modeling parameters in IMRT plan optimization

Keywords: intensity-modulated radiotherapy (IMRT) inverse IMRT planning, convex optimization, sensitivity analysis, elasticity, modeling parameters, equivalent uniform dose (EUD)

(18 pages, 2007)

\section{A. Wiegmann}

Computation of the permeability of porous materials from their microstructure by FFFStokes

Keywords: permeability, numerical homogenization

fast Stokes solver

(24 pages, 2007)

130. T. Melo, S. Nickel, F. Saldanha da Gama Facility Location and Supply Chain Management - A comprehensive review

Keywords: facility location, supply chain management, network design

(54 pages, 2007)

131. T. Hanne, T. Melo, S. Nickel

Bringing robustness to patient flow management through optimized patient transports in hospitals

Keywords: Dial-a-Ride problem, online problem, case study tabu search, hospital logistics

(23 pages, 2007)

132. R. Ewing, O. Iliev, R. Lazarov, I. Rybak, J. Willems

An efficient approach for upscaling properties of composite materials with high contrast of coefficients

Keywords: effective heat conductivity, permeability of fractured porous media, numerical upscaling, fibrous insulation materials, metal foams

(16 pages, 2008)

133. S. Gelareh, S. Nickel

New approaches to hub location problems in public transport planning Keywords: integer programming, hub location, transportation, decomposition, heuristic

(25 pages, 2008)

134. G. Thömmes, J. Becker, M. Junk, A. K. Vaikuntam, D. Kehrwald, A. Klar, K. Steiner, A. Wiegmann

A Lattice Boltzmann Method for immiscible multiphase flow simulations using the Level Set Method

Keywords: Lattice Boltzmann method, Level Set method, free surface, multiphase flow (28 pages, 2008

\section{J. Orlik}

Homogenization in elasto-plasticity

Keywords: multiscale structures, asymptotic homogenization, nonlinear energy

(40 pages, 2008)

136. J. Almquist, H. Schmidt, P. Lang, J. Deitmer, M. Jirstrand, D. Prätzel-Wolters, H. Becker

Determination of interaction between MCT1 and CAII via a mathematical and physiological approach

Keywords: mathematical modeling; model reduction: electrophysiology; $\mathrm{pH}$-sensitive microelectrodes; proton antenna

(20 pages, 2008)
137. E. Savenkov, H. Andrä, O. Iliev

An analysis of one regularization approach for solution of pure Neumann problem Keywords: pure Neumann problem, elasticity, regularization, finite element method, condition number (27 pages, 2008)

138. O. Berman, J. Kalcsics, D. Krass, S. Nickel The ordered gradual covering location problem on a network

Keywords: gradual covering, ordered median function, network location

(32 pages, 2008)

139. S. Gelareh, S. Nickel

Multi-period public transport design: A novel model and solution approaches Keywords: Integer programming, hub location, public transport, multi-period planning, heuristics (31 pages, 2008)

140. T. Melo, S. Nickel, F. Saldanha-da-Gama Network design decisions in supply chain planning

Keywords: supply chain design, integer programming models, location models, heuristics

(20 pages, 2008)

141. C. Lautensack, A. Särkkä, J. Freitag K. Schladitz

Anisotropy analysis of pressed point processes

Keywords: estimation of compression, isotropy test nearest neighbour distance, orientation analysis, polar ice, Ripley's K function

(35 pages, 2008)

142. O. Iliev, R. Lazarov, J. Willems

A Graph-Laplacian approach for calculating the effective thermal conductivity of complicated fiber geometries

Keywords: graph laplacian, effective heat conductivity, numerical upscaling, fibrous materials

(14 pages, 2008)

143. J. Linn, T. Stephan, J. Carlsson, R. Bohlin Fast simulation of quasistatic rod deformations for VR applications

Keywords: quasistatic deformations, geometrically exact rod models, variational formulation, energy minimization, finite differences, nonlinear conjugate gradients

(7 pages, 2008)

\section{J. Linn, T. Stephan}

Simulation of quasistatic deformations using discrete rod models

Keywords: quasistatic deformations, geometrically exact rod models, variational formulation, energy minimization, finite differences, nonlinear conjugate gradients

(9 pages, 2008

145. J. Marburger, N. Marheineke, R. Pinnau Adjoint based optimal control using meshless discretizations

Keywords: Mesh-less methods, particle methods, Eulerian-Lagrangian formulation, optimization strategies, adjoint method, hyperbolic equations

(14 pages, 2008

\section{S. Desmettre, J. Gould, A. Szimayer}

Own-company stockholding and work effort preferences of an unconstrained executive Keywords: optimal portfolio choice, executive compensation

(33 pages, 2008) 
147. M. Berger, M. Schröder, K.-H. Küfer A constraint programming approach for the two-dimensional rectangular packing problem with orthogonal orientations

Keywords: rectangular packing, orthogonal orientations non-overlapping constraints, constraint propagation

(13 pages, 2008)

148. K. Schladitz, C. Redenbach, T. Sych, M. Godehardt

Microstructural characterisation of open foams using $3 d$ images

Keywords: virtual material design, image analysis, open foams

(30 pages, 2008)

149. E. Fernández, J. Kalcsics, S. Nickel, R. Ríos-Mercado

A novel territory design model arising in the implementation of the WEEE-Directive Keywords: heuristics, optimization, logistics, recycling (28 pages, 2008)

150. H. Lang, J. Linn

Lagrangian field theory in space-time for geometrically exact Cosserat rods Keywords: Cosserat rods, geometrically exact rods, small strain, large deformation, deformable bodies, Lagrangian field theory, variational calculus (19 pages, 2009)

151. K. Dreßler, M. Speckert, R. Müller, Ch. Weber

Customer loads correlation in truck engineering

Keywords: Customer distribution, safety critical components, quantile estimation, Monte-Carlo methods (11 pages, 2009)

152. H. Lang, K. Dreßler

An improved multiaxial stress-strain correction model for elastic FE postprocessing Keywords: Jiang's model of elastoplasticity, stress-strain correction, parameter identification, automatic differentiation, least-squares optimization, Coleman-Li algorithm

(6 pages, 2009)

153. J. Kalcsics, S. Nickel, M. Schröder A generic geometric approach to territory design and districting

Keywords: Territory design, districting, combinatorial optimization, heuristics, computational geometry (32 pages, 2009)

154. Th. Fütterer, A. Klar, R. Wegener An energy conserving numerical scheme for the dynamics of hyperelastic rods

Keywords: Cosserat rod, hyperealstic, energy conservation, finite differences

(16 pages, 2009)

155. A. Wiegmann, L. Cheng, E. Glatt, O. Iliev, S. Rief

Design of pleated filters by computer simulations

Keywords: Solid-gas separation, solid-liquid separation, pleated filter, design, simulation

(21 pages, 2009)

156. A. Klar, N. Marheineke, R. Wegener Hierarchy of mathematical models for production processes of technical textiles
Keywords: Fiber-fluid interaction, slender-body theory, turbulence modeling, model reduction, stochastic differential equations, Fokker-Planck equation, asymptotic expansions, parameter identification

(21 pages, 2009)

157. E. Glatt, S. Rief, A. Wiegmann, M. Knefel, E. Wegenke

Structure and pressure drop of real and virtual metal wire meshes

Keywords: metal wire mesh, structure simulation, model calibration, CFD simulation, pressure loss (7 pages, 2009)

\section{S. Kruse, M. Mülle}

Pricing American call options under the assumption of stochastic dividends - An application of the Korn-Rogers model Keywords: option pricing, American options, dividends, dividend discount model, Black-Scholes model (22 pages, 2009)

159. H. Lang, J. Linn, M. Arnold

Multibody dynamics simulation of geometrically exact Cosserat rods

Keywords: flexible multibody dynamics, large deformations, finite rotations, constrained mechanical systems, structural dynamics

(20 pages, 2009)

160. P. Jung, S. Leyendecker, J. Linn, M. Ortiz Discrete Lagrangian mechanics and geometrically exact Cosserat rods

Keywords: special Cosserat rods, Lagrangian mechanics, Noether's theorem, discrete mechanics, frame-indifference, holonomic constraints

(14 pages, 2009)

161. M. Burger, K. Dreßler, A. Marquardt, M. Speckert

Calculating invariant loads for system simulation in vehicle engineering

Keywords: iterative learning control, optimal control

theory, differential algebraic equations (DAEs)

(18 pages, 2009)

162. M. Speckert, N. Ruf, K. Dreßler Undesired drift of multibody models excited by measured accelerations or forces Keywords: multibody simulation, full vehicle model, force-based simulation, drift due to noise

(19 pages, 2009)

163. A. Streit, K. Dreßler, M. Speckert, J. Lichter, T. Zenner, P. Bach

Anwendung statistischer Methoden zur Erstellung von Nutzungsprofilen für die Auslegung von Mobilbaggern

Keywords: Nutzungsvielfalt, Kundenbeanspruchung, Bemessungsgrundlagen

(13 pages, 2009)

164. I. Correia, S. Nickel, F. Saldanha-da-Gama The capacitated single-allocation hub location problem revisited: A note on a classical formulation

Keywords: Capacitated Hub Location, MIP formulations (10 pages, 2009)

165. F. Yaneva, T. Grebe, A. Scherrer

An alternative view on global radiotherapy optimization problems

Keywords: radiotherapy planning, path-connected sublevelsets, modified gradient projection method, improving and feasible directions

(14 pages, 2009)
166. J. I. Serna, M. Monz, K.-H. Küfer, C. Thieke Trade-off bounds and their effect in multicriteria IMRT planning

Keywords: trade-off bounds, multi-criteria optimization, IMRT, Pareto surface

(15 pages, 2009)

167. W. Arne, N. Marheineke, A. Meister, R. Wegener

Numerical analysis of Cosserat rod and string models for viscous jets in rotational spinning processes

Keywords: Rotational spinning process, curved viscous fibers, asymptotic Cosserat models, boundary value problem, existence of numerical solutions

(18 pages, 2009)

168. T. Melo, S. Nickel, F. Saldanha-da-Gama An LP-rounding heuristic to solve a multiperiod facility relocation problem

Keywords: supply chain design, heuristic, linear programming, rounding

(37 pages, 2009)

169. I. Correia, S. Nickel, F. Saldanha-da-Gama Single-allocation hub location problems with capacity choices

Keywords: hub location, capacity decisions, MILP formulations

(27 pages, 2009)

170. S. Acar, K. Natcheva-Acar A guide on the implementation of the Heath-Jarrow-Morton Two-Factor Gaussian Short Rate Model (HJM-G2++)

Keywords: short rate model, two factor Gaussian, G2++, option pricing, calibration

(30 pages, 2009)

171. A. Szimayer, G. Dimitroff, S. Lorenz A parsimonious multi-asset Heston model: calibration and derivative pricing Keywords: Heston model, multi-asset, option pricing, calibration, correlation

(28 pages, 2009)

172. N. Marheineke, R. Wegener Modeling and validation of a stochastic drag for fibers in turbulent flows Keywords: fiber-fluid interactions, long slender fibers, turbulence modelling, aerodynamic drag, dimensional analysis, data interpolation, stochastic partial differential algebraic equation, numerical simulations, experimental validations

(19 pages, 2009)

173. S. Nickel, M. Schröder, J. Steeg Planning for home health care services Keywords: home health care, route planning, metaheuristics, constraint programming

(23 pages, 2009)

174. G. Dimitroff, A. Szimayer, A. Wagner Quanto option pricing in the parsimonious Heston model

Keywords: Heston model, multi asset, quanto options, option pricing

(14 pages, 2009) 174. G. Dimitroff, A. Szimayer, A. Wagner

175. S. Herkt, K. Dreßler, R. Pinnau

Model reduction of nonlinear problems in structural mechanics

Keywords: flexible bodies, FEM, nonlinear model reduc tion, $P O D$

(13 pages, 2009) 
176. M. K. Ahmad, S. Didas, J. Iqbal

Using the Sharp Operator for edge detection and nonlinear diffusion

Keywords: maximal function, sharp function, image processing, edge detection, nonlinear diffusion

(17 pages, 2009)

177. M. Speckert, N. Ruf, K. Dreßler, R. Müller, C. Weber, S. Weihe

Ein neuer Ansatz zur Ermittlung von Erprobungslasten für sicherheitsrelevante Bauteile

Keywords: sicherheitsrelevante Bauteile, Kundenbeanspruchung, Festigkeitsverteilung, Ausfallwahrscheinlichkeit, Konfidenz, statistische Unsicherheit, Sicherheitsfaktoren

(16 pages, 2009)

\section{J. Jegorovs}

Wave based method: new applicability areas Keywords: Elliptic boundary value problems, inhomogeneous Helmholtz type differential equations in bounded domains, numerical methods, wave based method, uniform B-splines

(10 pages, 2009)

179. H. Lang, M. Arnold

Numerical aspects in the dynamic simulation of geometrically exact rods Keywords: Kirchhoff and Cosserat rods, geometrically exact rods, deformable bodies, multibody dynamics, artial differential algebraic equations, method of lines, time integration

(21 pages, 2009)

180. H. Lang

Comparison of quaternionic and rotationfree null space formalisms for multibody dynamics

Keywords: Parametrisation of rotations, differentialalgebraic equations, multibody dynamics, constrained mechanical systems, Lagrangian mechanics

(40 pages, 2010)

181. S. Nickel, F. Saldanha-da-Gama, H.-P. Ziegler Stochastic programming approaches for risk aware supply chain network design problems Keywords: Supply Chain Management, multi-stage stochastic programming, financial decisions, risk (37 pages, 2010)

\section{P. Ruckdeschel, N. Horbenko}

Robustness properties of estimators in generalized Pareto Models

Keywords: global robustness, local robustness, finite sample breakdown point, generalized Pareto distribution (58 pages, 2010)

\section{P. Jung, S. Leyendecker, J. Linn, M. Ortiz}

A discrete mechanics approach to Cosserat rod theory - Part 1: static equilibria

Keywords: Special Cosserat rods; Lagrangian mechanics; Noether's theorem; discrete mechanics; frameindifference; holonomic constraints; variational formulation

(35 pages, 2010)

184. R. Eymard, G. Printsypar

A proof of convergence of a finite volume scheme for modified steady Richards' equation describing transport processes in the pressing section of a paper machine Keywords: flow in porous media, steady Richards' equation, finite volume methods, convergence of approximate solution

(14 pages, 2010)

185. P. Ruckdeschel

\section{Optimally Robust Kalman Filtering}

Keywords: robustness, Kalman Filter, innovation outlier, additive outlier

(42 pages, 2010)

186. S. Repke, N. Marheineke, R. Pinnau On adjoint-based optimization of a free surface Stokes flow

Keywords: film casting process, thin films, free surface Stokes flow, optimal control, Lagrange formalism (13 pages, 2010)

187. O. Iliev, R. Lazarov, J. Willems

Variational multiscale Finite Element Method for flows in highly porous media Keywords: numerical upscaling, flow in heterogeneous porous media, Brinkman equations, Darcy's law, subgrid approximation, discontinuous Galerkin mixed FEM (21 pages, 2010)

\section{S. Desmettre, A. Szimayer}

Work effort, consumption, and portfolio selection: When the occupational choice matters

Keywords: portfolio choice, work effort, consumption, occupational choice

(34 pages, 2010)

189. O. Iliev, Z. Lakdawala, V. Starikovicius On a numerical subgrid upscaling algorithm for Stokes-Brinkman equations

Keywords: Stokes-Brinkman equations, subgrid approach, multiscale problems, numerical upscaling (27 pages, 2010)

190. A. Latz, J. Zausch, O. Iliev

Modeling of species and charge transport in Li-Ion Batteries based on non-equilibrium thermodynamics

Keywords: lithium-ion battery, battery modeling, electrochemical simulation, concentrated electrolyte, ion transpor

(8 pages, 2010)

191. P. Popov, Y. Vutov, S. Margenov, O. Iliev Finite volume discretization of equations describing nonlinear diffusion in Li-Ion batteries

Keywords: nonlinear diffusion, finite volume discretization, Newton method, Li-lon batteries

(9 pages, 2010)

192. W. Arne, N. Marheineke, R. Wegener Asymptotic transition from Cosserat rod to string models for curved viscous inertial jets

Keywords: rotational spinning processes; inertial and viscous-inertial fiber regimes; asymptotic limits; slenderbody theory; boundary value problems

(23 pages, 2010)

193. L. Engelhardt, M. Burger, G. Bitsch Real-time simulation of multibody-systems for on-board applications

Keywords: multibody system simulation, real-time simulation, on-board simulation, Rosenbrock methods (10 pages, 2010)

\section{M. Burger, M. Speckert, K. Dreßler} Optimal control methods for the calculation of invariant excitation signals for multibody systems

Keywords: optimal control, optimization, mbs simulation, invariant excitation

(9 pages, 2010)
195. A Latz, J. Zausch

Thermodynamic consistent transport theory of Li-lon batteries

Keywords: Li-lon batteries, nonequilibrium thermodynamics, thermal transport, modeling

(18 pages, 2010)

\section{S. Desmettre}

Optimal investment for executive stockholders with exponential utility Keywords: portfolio choice, executive stockholder work effort, exponential utility

(24 pages, 2010)

197. W. Arne, N. Marheineke, J. Schnebele, R. Wegener

Fluid-fiber-interactions in rotational spinning process of glass wool production Keywords: Rotational spinning process, viscous thermal jets, fluid-fiber-interactions, two-way coupling, slenderbody theory, Cosserat rods, drag models, boundary value problem, continuation method

(20 pages, 2010)

198. A. Klar, J. Maringer, R. Wegener

A 3d model for fiber lay-down in nonwoven production processes

Keywords: fiber dynamics, Fokker-Planck equations, diffusion limits

(15 pages, 2010)

199. Ch. Erlwein, M. Müller

A regime-switching regression model for hedge funds

Keywords: switching regression model, Hedge funds, optimal parameter estimation, filtering

(26 pages, 2011)

200. M. Dalheimer

Power to the people - Das Stromnetz der Zukunft

Keywords: Smart Grid, Stromnetz, Erneuerbare Energien, Demand-Side Management

(27 pages, 2011)

201. D. Stahl, J. Hauth

PF-MPC: Particle Filter-Model Predictive Control

Keywords: Model Predictive Control, Particle Filter, CSTR, Inverted Pendulum, Nonlinear Systems, Sequential Monte Carlo

(40 pages, 2011)

\section{G. Dimitroff, J. de Kock}

Calibrating and completing the volatility cube in the SABR Model

Keywords: stochastic volatility, SABR, volatility cube, swaption

(12 pages, 2011)

203. J.-P. Kreiss, T. Zangmeister

Quantification of the effectiveness of a safety function in passenger vehicles on the basis of real-world accident data Keywords: logistic regression, safety function, realworld accident data, statistical modeling (23 pages, 2011)

204. P. Ruckdeschel, T. Sayer, A. Szimayer Pricing American options in the Heston model: a close look on incorporating correlation

Keywords: Heston model, American options, moment matching, correlation, tree method

(30 pages, 2011) 
205. H. Ackermann, H. Ewe, K.-H. Küfer,

M. Schröder

Modeling profit sharing in combinatorial exchanges by network flows

Keywords: Algorithmic game theory, profit sharing, combinatorial exchange, network flows, budget balance, core

(17 pages, 2011)

206. O. lliev, G. Printsypar, S. Rief

A one-dimensional model of the pressing section of a paper machine including $d y$ namic capillary effects

Keywords: steady modified Richards' equation, finite volume method, dynamic capillary pressure, pressing section of a paper machine

(29 pages, 2011)

207. I. Vecchio, K. Schladitz, M. Godehardt,

M. J. Heneka

Geometric characterization of particles in $3 d$ with an application to technical cleanliness

Keywords: intrinsic volumes, isoperimetric shape factors, bounding box, elongation, geodesic distance, technical cleanliness

(21 pages, 2011)

208. M. Burger, K. Dreßler, M. Speckert Invariant input loads for full vehicle multibody system simulation

Keywords: multibody systems, full-vehicle simulation, optimal control

(8 pages, 2011)

209. H. Lang, J. Linn, M. Arnold

Multibody dynamics simulation of geometrically exact Cosserat rods

Keywords: flexible multibody dynamics, large deformations, finite rotations, constrained mechanical systems, structural dynamics

(28 pages, 2011)

210. G. Printsypar, R. Ciegis

On convergence of a discrete problem describing transport processes in the pressing section of a paper machine including dynamic capillary effects: one-dimensional case

Keywords: saturated and unsaturated fluid flow in porous media, Richards' approach, dynamic capillary pressure, finite volume methods, convergence of approximate solution

(24 pages, 2011)

211. O. Iliev, G. Printsypar, S. Rief

A two-cimensional model of the pressing section of a paper machine including $d y$ namic capillary effects

Keywords: two-phase flow in porous media, steady modified Richards' equation, finite volume method, $d y$ namic capillary pressure, pressing section of a paper machine, multipoint flux approximation

(44 pages, 2012) 
